\title{
Analytical Models for the Response of the Double-Bottom Structure to Underwater Explosion Based on the Wave Motion Theory
}

\author{
Yingyu Chen, ${ }^{1}$ Xiongliang Yao, ${ }^{1}$ and Wei Xiao ${ }^{2}$ \\ ${ }^{1}$ College of Shipbuilding Engineering, Harbin Engineering University, Harbin 150001, China \\ ${ }^{2}$ College of Engineering, Ocean University of China, Qingdao 266100, China \\ Correspondence should be addressed to Xiongliang Yao; xiongliangyao@gmail.com
}

Received 26 December 2015; Revised 18 April 2016; Accepted 8 May 2016

Academic Editor: Lorenzo Dozio

Copyright (C) 2016 Yingyu Chen et al. This is an open access article distributed under the Creative Commons Attribution License, which permits unrestricted use, distribution, and reproduction in any medium, provided the original work is properly cited.

\begin{abstract}
The aim of this paper is to apply the elastic wave motion theory and the classical one-dimensional cavitation theory to analyze the response of a typical double-bottom structure subjected to underwater blast. The section-varying bar theory and the general acoustic impedance are introduced to get the simplified analytical models. The double-bottom structure is idealized by the basic unit of three substructures which include the simple panel, the panel with stiffener (T-shaped), and the panel associated with girder (I-shaped). According to the simplified models, the analytical models for the corresponding substructures are set up. By taking the cavitation effect into account, the process of fluid-structure interaction can be thoroughly understood, as well as the stress wave propagation. Good agreement between the analytical solution and the finite element prediction is achieved. On the other hand, the Taylor predictions for the panel associated with girder (I-shaped) including the effects of cavitation are invalid, indicating a potential field for the analytical method. The validated analytical models are used to determine the sensitivity of structure response to dimensionless geometric parameters $\bar{\alpha}^{(k)}, \bar{\beta}^{(k)}$, and $\bar{\gamma}^{(k)}$. Based on the dynamic response of the substructures, we establish the approximate analytical models which are able to predict the response of double-bottom structure to underwater explosion.
\end{abstract}

\section{Introduction}

A considerable amount of literature exists on the feature of underwater explosion load and the dynamic response of ship structure subjected to underwater explosion [1-7]. The dynamic response of double-bottom hull to underwater explosion is of great interest to the defense of military naval vessels. In order to obtain the dynamic response of doublebottom hull, the effects of fluid-structure interaction (FSI) during the loading phase need to be thoroughly understood. The effects of FSI include two aspects: the shock wave propagation in the complex structure and the cavitation effects in the liquid surrounding the concerned structure.

First theoretical studies on FSI date back to World War II. Taylor [8] investigated the response of the free-standing air-backed rigid plate subjected to water blast and found the impulse transmitted to the plate could be reduced by reducing the mass of the plate, but the reduction leads to water cavitation at the fluid-structure interface. The development of Taylor's solution has been done to investigate the response of protective structures [4, 9-12]. When the input wave acts on the fluid-structure interface, the stress wave propagates along the longitudinal direction in structure [13]. By taking longitudinal stress waves into consideration, Jin et al. [14, 15] proposed an analytical model to solve the response of a coated plate to water blast. However, these methods cannot address the cavitation effects.

The loads due to cavitation need to be taken into consideration. By treating water as a bilinear elastic medium, Kennard [16] theoretically studied the one-dimensional cavitation phenomena consequent to the shock wave and described the propagating process of the "breaking front" and the "closing front." Schiffer et al. [4] analytically investigated the onedimensional response of a spring-supported rigid plate to 
underwater blast. They focused on the cavitation phenomena in the fluid, including propagation and arrest of breaking fronts and closing fronts. Later on, the structure response and the cavitation process subsequent to underwater blast were experimentally investigated [17-19]. It was found that the breaking front approaching the structure may invert its direction before reaching the fluid-structure interface and the behavior of water cavitation strongly influenced the pressure histories of the structure. Based on linear wave motion theory, Jin et al. [20] investigated a monolithic elastic coating with varying stiffness and thickness with the consideration of FSI effects and cavitation phenomena. The analytical solutions for propagation of breaking fronts and closing front as well as their interactions with the structure under a water blast were found to be in excellent agreement with finite element (FE) predictions.

A significant part of the recent literature on blast loading has concentrated on the underwater blast loading of sandwich structure [17, 21-25]. Deshpande and Fleck [21] and Hutchinson and Xue [22] proposed approximate analytical models for the one-dimensional response of sandwich plate subjected to underwater shock load. McMeeking et al. [25] developed an analytical model for wet surface response, including fluid-structure interaction, which can be used for a wide range of core topologies. This model addressed cavitation and incorporated the momentum of reconstituted water attached to the wet face. Yin et al. [11] approached a similar problem by performing FE simulations and developing analytical models. The results showed that the actual incident wave will be enhanced due to the collapse of cavitation bubbles, especially for the soft core.

Although considerable efforts have been devoted to understanding the effects of FSI on the 1D response of monolithic plates and sandwich panels, it still remains unclear how the wave propagation and FSI affect the response of a complex structure such as double-bottom structures. Analytical models for study of the double-bottom structures response are rare. When analyzing a tanker grounding accident, the total response of the assembly is obtained through the summation of the responses of all structural members [26, 27]: plates, cruciform, and stiffeners. Based on similar models, a potential alternative simplified calculation model for the total response of the double-bottom can be obtained in two steps. In the first step, we analytically solve the structural response for the individual structural members: the simple panel, the panel with stiffener (T-shaped), and the panel associated with girder (I-shaped). In the second step, the total response of the double-bottom structure can be obtained through the summation of the responses of the three structural members. Based on this, an effective and quick prediction of the response of double-bottom structure can be achieved.

In this study, we examined the dynamic response of the free-standing double-bottom structure plates in contact with a liquid on one side to underwater explosion. We proposed analytical models taking into consideration the stress wave propagation in the structure, the cavitation effects in the fluid, and the corresponding effects on the plates. The general acoustic impedance $[28,29]$ is adopted to model the shape effect in the panel with stiffener (T-shaped) and the panel
TABle 1: Parameter for the substructures (unit: $\mathrm{m}$ ).

\begin{tabular}{|c|c|c|c|c|c|c|}
\hline & $t_{f}$ & $t_{m}$ & $t_{b}$ & $l_{f}$ & $d_{m}$ & $l_{b}$ \\
\hline Simple panel & 0.01 & - & - & - & - & - \\
\hline $\begin{array}{l}\text { Panel with stiffener } \\
\text { (T-shaped) }\end{array}$ & 0.01 & 0.01 & - & 0.07 & 0.07 & - \\
\hline $\begin{array}{l}\text { Panel with girder } \\
\text { (I-shaped) }\end{array}$ & 0.01 & 0.01 & 0.01 & 0.07 & 0.8 & 0.07 \\
\hline
\end{tabular}

associated with girder (I-shaped). The theory of cavitation in Kennard's research $[4,16]$ is used to model the cavitation effect in the fluid. Based on the wave motion theory [15], the longitudinal stress waves are taken into account to obtain a more precise solution. One important difference between our approach and Jin et al.s work [14, 20] is that Jin's theory is usually employed in the q-layer system. We however use it to analyze the double-bottom structure dynamics by introducing the general acoustic impedance and established the relationship between the substructures dynamics and the double-bottom structure dynamics.

The outline of the paper is as follows: in Section 2, we present the analytical models for substructures; in Section 3, FE models for corresponding substructures are described and the comparisons between the analytical and FE predictions are carried out; in Section 4, by using the validated analytical models, we explore the sensitivity of the substructures response to different parameters; in Section 5, based on the analysis of substructures, we construct the approximate analytical solutions for a double-bottom structure.

\section{Analytical Models}

2.1. Model Characteristics. A typical double-bottom structure of a naval ship subjected to underwater explosion is shown in Figure 1(a). This structure is slightly idealized when compared to the real structure. The profile of longitudinal stiffener and girder is rectangular and the double-bottom structure's height is a constant. The stress waves propagating process in the double-bottom structure hull is rather complex. To achieve a simple and effective model, we divide the whole model into three substructures (see Figure 1(b)): the panel (base component of the ship's hull structure), a panel with longitudinal stiffener (T-shaped), and girders (I-shaped).

The profiles for the panel, the panel with stiffener (Tshaped), and the panel with girder (I-shaped) are shown in Figure 2 and Table 1 . The considered variables are dimensions of profile element's width and thickness of the bottom flange $l_{f}, t_{f}$, the web $d_{m}, t_{m}$, and the top flange $l_{b}, t_{b}$. The profile was characterized with dimensionless geometric parameter $l_{f} / t_{m}, d_{m} / t_{f}, l_{b} / t_{m}, d_{m} / l_{f}$. The distance between two girders is $1 \mathrm{~m}$.

Due to the varying cross section of the structure, the stress wave will be redistributed since a portion will be reflected and the other will be transmitted at the interface (see Figure 3 ). $\rho_{i}, c_{i}$, and $A_{i}$ are the density, sound speed, and the area of the section of the medium $i, i=1,2$, respectively. The medium on the left side of the interface is taken as medium 1 and the other side is medium 2. Therefore, the subscripts of the variables 


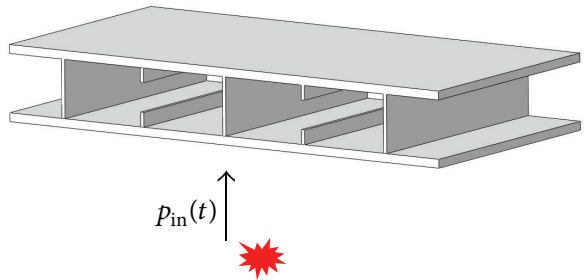

(a)

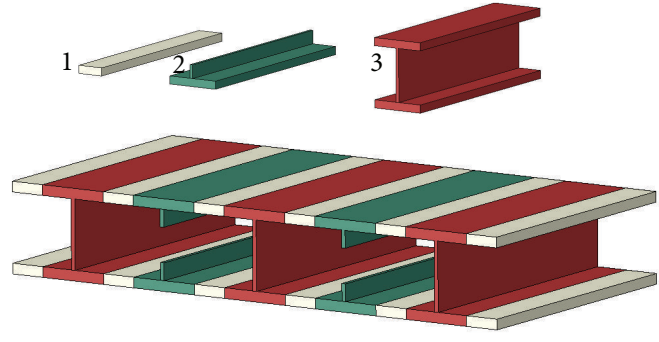

(b)

FiguRE 1: Sketches of problem geometry for double-bottom structure: (a) typical double-bottom structure to underwater blast; (b) three substructures for the double-bottom structure.

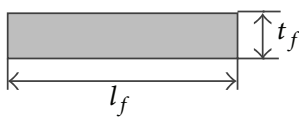

(a) Panel

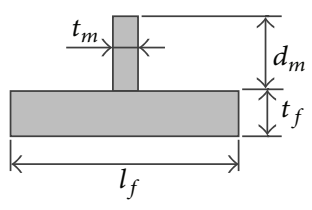

(b) T-shaped plate

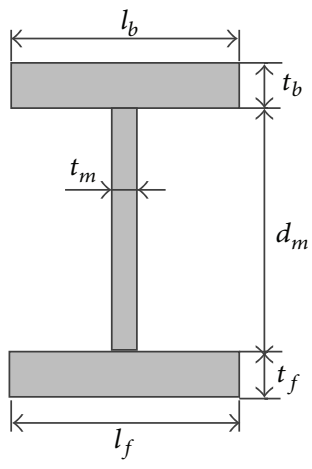

(c) I-shaped plate

FIGURE 2: The geometries of the three substructures used in this analysis.

refer to medium 1 and medium 2, for example, $\rho_{1} c_{1} A_{1}$ and $\rho_{2} \mathcal{C}_{2} A_{2} . \Delta \sigma_{I}, \Delta \sigma_{R}$, and $\Delta \sigma_{T}$ are the disturbance quantity of stress of incident wave, reflected wave, and transmitted wave, respectively.

According to the force equilibrium and compatibility at the interface, we can obtain

$$
\begin{aligned}
A_{1}\left(\Delta \sigma_{I}+\Delta \sigma_{R}\right) & =A_{2}\left(\Delta \sigma_{T}\right), \\
\frac{\Delta \sigma_{I}}{\rho_{1} c_{1}}-\frac{\Delta \sigma_{R}}{\rho_{1} c_{1}} & =\frac{\Delta \sigma_{T}}{\rho_{2} c_{2}} .
\end{aligned}
$$

By denoting the ratio of general acoustic impedance $n=$ $\left(\rho_{1} c_{1} A_{1}\right) /\left(\rho_{2} c_{2} A_{2}\right),(1)$ can be rewritten as

$$
\begin{aligned}
& \Delta \sigma_{R}=r \cdot \Delta \sigma_{I}, \quad r=\frac{1-n}{1+n}, \\
& \Delta \sigma_{T}=s \cdot \Delta \sigma_{I}, \quad s=\frac{2}{1+n} \cdot \frac{A_{1}}{A_{2}},
\end{aligned}
$$

where $r$ is the reflection coefficient and $s$ is the transmission coefficient.

In this paper, the general acoustic impedance [28] is introduced to consider the varying section in the structure. Based on this, we can assume that substructures in doublebottom structure subjected to underwater explosion are treated as one-dimensional multilayer system structures to water blast. The analytical method described in [14] is used

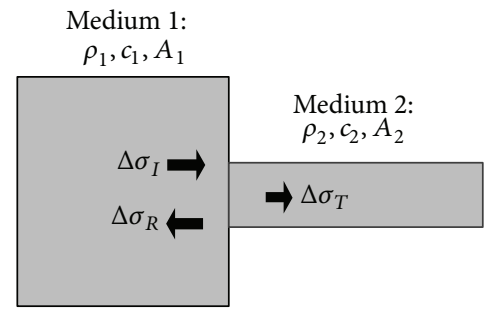

FIGURE 3: The wave reflection and transmission at the interface.

to analyze the stress wave propagation in this studies. The following assumptions are made: the substructures (panel, the panel with stiffener (T-shaped), and the panel with girder (I-shaped)) are treated as multilayer medium with different general acoustic impedance; the pressure wave travels as a longitudinal wave; and the rigid body motion is neglected.

Underwater explosions give rise to spherical shockwaves, traveling in water at approximately sonic speed and impinging on structures. At sufficient distance from the point of denotation, spherical shockwaves can be taken as onedimensional planar wave. Assume that the double-bottom structure is loaded by a planar, exponentially decaying pressure wave with the peak pressure $p_{0}$ and decay constant $\theta$, traveling towards the structure. Based on the assumptions we listed above, to thoroughly understand the fluid-structure 


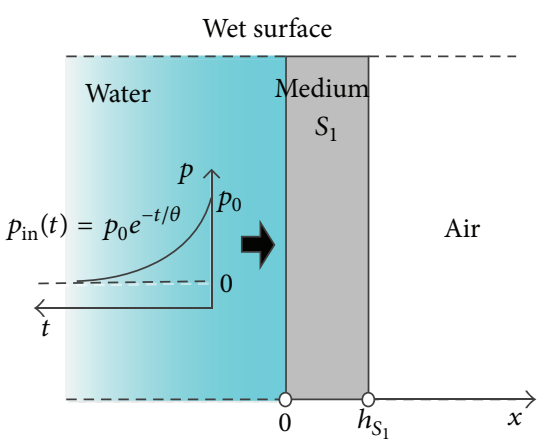

(a)

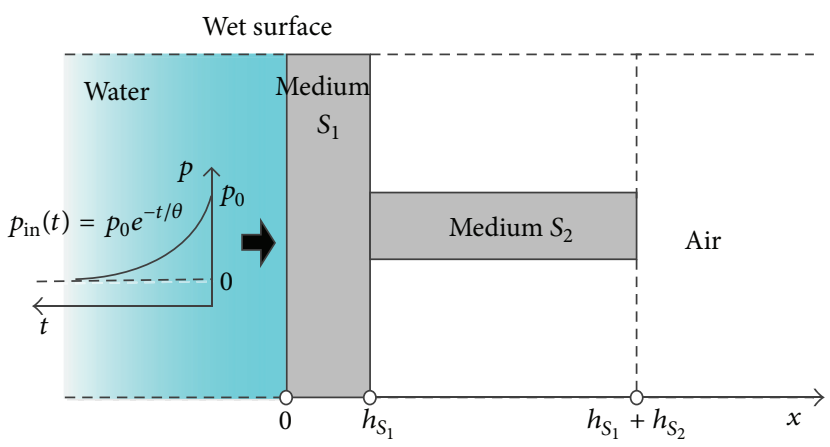

(b)

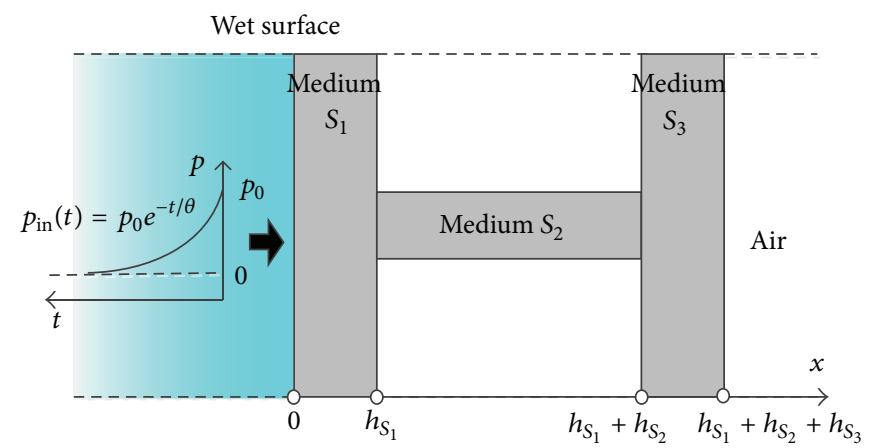

(c)

FIGURE 4: Sketches of geometry, reference system, and loading case for (a) the simple panel; (b) the panel with stiffener (T-shaped); (c) the panel with girder (I-shaped).

interaction, we proceed to derive the double-bottom structure response for three cases sketched in Figure 4 and described as follows:

(1) A steel panel plate with one medium (mediums $S_{1}$ ) is in contact with water (see Figure 4(a)).

(2) A panel with stiffener (T-shaped) is treated as a structure with two mediums (mediums $S_{1}$ and $S_{2}$ ) and is in contact with water (see Figure 4(b)).

(3) An panel with girder (I-shaped) is treated as a structure with three mediums (mediums $S_{1}, S_{2}$, and $S_{3}$ ) and is in contact with water (see Figure $4(\mathrm{c})$ ).

2.2. Fluid-Structure Interaction. According to the reference system shown in Figure 4, waves traveling in the positive $x$ direction at an arbitrary time $t$ can be expressed as

$$
p_{\text {in }}(x, t)=p_{0} e^{-\left(t-x / c_{w}\right) / \theta},
$$

where $p_{0}$ is the peak pressure and $\theta$ is the decay constant. Applying the continuity of the pressure and particle velocity on the wet surface, we can obtain

$$
\begin{aligned}
p_{\text {in }}(t)+p_{\text {ref }}(t) & =p_{\text {tra }}(t), \\
\frac{p_{\text {in }}(t)}{\rho_{w} c_{w}}-\frac{p_{\text {ref }}(t)}{\rho_{w} c_{w}} & =\frac{p_{\text {tra }}(t)}{\rho_{s} c_{s}},
\end{aligned}
$$

where $\rho_{w}$ is the water density, $c_{w}$ is the sonic speed of water, and $\rho_{s}$ and $c_{s}$ are the density and sonic speed of the structure. $p_{\text {in }}(t), p_{\text {ref }}(t)$, and $p_{\text {tra }}(t)$ represent the pressure induced by incident wave, reflected wave, and transmitted wave on the wet surface, respectively.

In this paper, we assume that the water cavitation pressure is zero, in line with the assumptions of previous studies in this field $[18,25,30]$. In order to clarify the interaction process, we divide the response process into two stages: (i) the response prior to the onset of first cavitation and (ii) the response subsequent to the first cavitation.

\subsubsection{Response of Three Substructures prior to the Onset of First} Cavitation. We would like to reemphasize one important difference between our studying and that of Jin et al's work [15]. Jin's theory is usually employed in multilayer system. We developed a method based on Jin's work to analyze the double-bottom structure dynamics by introducing the general acoustic impedance and established the analytical models between the substructures and the double-bottom structure.

(a) Response of the Simple Panel. Consider the problem sketched in Figure 4(a) of a plate in contact with water on the left side. Let us define $t_{2 S_{1}}=2 h_{S_{1}} / c_{S_{1}}$ as the time the wave front propagates through a plate with thickness $h_{S_{1}}$ and back to the wet surface. Assume that the stress waves have been propagated through the steel plates $m_{1}$ times. Thus, $t_{m_{1}}$ can be expressed as

$$
t_{m_{1}}=m_{1} \cdot t_{2 S_{1}},
$$




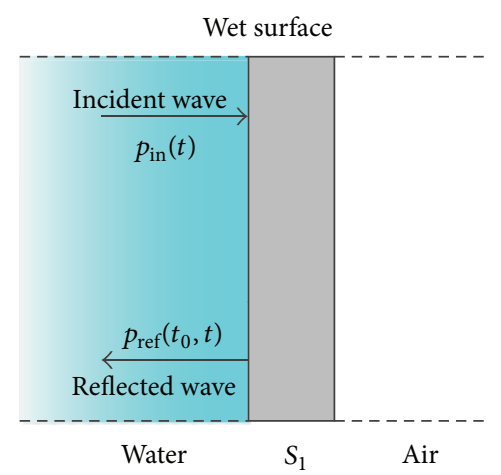

(a) $m_{1}=0$

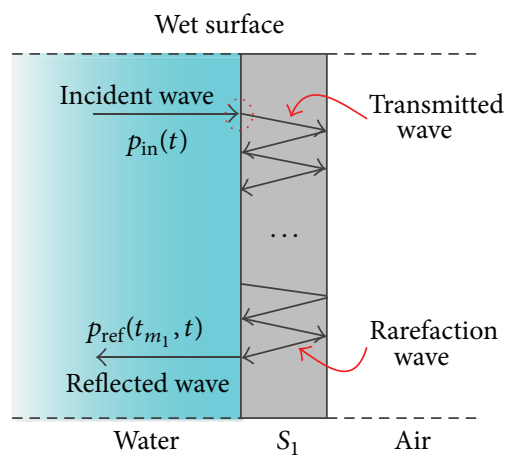

(b) $m_{1}>0$

FIGURE 5: The wave propagation process in the panel: (a) the wave not transmitted into the steel plate (b) the wave transmitted into steel plate once or more.

where $m_{1}$ can be taken from 0 to infinity. Let $t_{-}$total be the total calculation time; then, $m_{1}$ means the value of $t_{-}$total $/ t_{2 S}$ rounded to the nearest integer. If $m_{1}=0$, it means that the wave is not transmitted into the steel plate, as shown in Figure 5(a), but it is reflected. If $m_{1} \geq 1$, it means that the input wave is transmitted into the steel plate and propagates $m_{1}$ times in steel plate, as shown in Figure 5(b).

Therefore, the reflection pressure on the wet surface can be expressed as

$$
\begin{aligned}
& p_{\text {ref }, m_{1}}^{(1)}\left(t_{m_{1}}, t\right) \\
& = \begin{cases}r_{w s_{1}} \cdot p_{\text {in }}(t), & \text { for } m_{1}=0, \\
s_{w s_{1}} s_{s_{1} w}\left(r_{s_{1} w} r_{s_{1}} a\right)^{m_{1}-1} r_{s_{1}} \cdot p_{\text {in }}\left(t-t_{m_{1}}\right), & \text { for } m_{1}>1,\end{cases} \\
& p_{\text {ref }}^{(1)}(t)=\sum_{i=0}^{m_{1}} p_{\text {ref }, i}^{(1)}\left(t_{i}, t\right) .
\end{aligned}
$$

Here, the subscript $w s_{1}$ represents the quantity from water to steel plate and $s_{1} w$ indicates the quantity from steel plate to water. Namely, $w$ refers to water, $s_{1}$ refers to the steel plate for only one medium, and $a$ is for air. $r_{w s_{1}}$ and $s_{w s_{1}}$ are the reflected coefficient and transmitted coefficient from water to steel plate, respectively. The ratio of two different general acoustic impedance mediums is denoted by $n_{w s_{1}}=$ $\left(\rho_{w} c_{w} A_{w}\right) /\left(\rho_{s} c_{s} A_{s}\right)$. Then, we have $r_{w s_{1}}=\left(1-n_{w s_{1}}\right) /\left(1+n_{w s_{1}}\right)$ and $s_{w s_{1}}=2\left(A_{w} / A_{s_{1}}\right) /\left(1+n_{w s_{1}}\right)$.

(b) Response of the Panel with Stiffener (T-Shaped). In Figure 4(b), we define $t_{2 S_{1}}$ as the time the input wave propagates once in medium $S_{1}$ and defined $t_{2 S_{2}}$ as the time the input wave propagates one time in medium $S_{2}$. $h_{S_{1}}$ and $h_{S_{2}}$ are the thickness of medium $S_{1}$ and medium $S_{2}$. Then, we have the $t_{2 S_{1}}=2 h_{S_{1}} / c_{S_{1}}$, and $t_{2 S_{2}}=2 h_{S_{2}} / c_{S_{2}}$. Thus, the specific time $t_{m_{1}, m_{2}}$ can be expressed as

$$
t_{m_{1}, m_{2}}=m_{1} \cdot t_{2 S_{1}}+m_{2} \cdot t_{2 S_{2}} .
$$

The value of $m_{1}$ and $m_{2}$ can be between 0 and infinity. Let $t_{-}$total be the total calculation time; then, $m_{1}$ means the value of $t_{-}$total $/ t_{2 S_{1}}$ rounded to the nearest integer and $m_{2}$ means the value of $t_{-}$total $/ t_{2 S_{2}}$ rounded to the nearest integer. Different cases are shown in Figure 6. If $m_{1}=m_{2}=0$, it indicates that the input wave is not transmitted into medium $S_{1}$. If $m_{1} \geq 1$ and $m_{2}=0$, the wave transmits from water into medium $S_{1}$ and propagates $m_{1}$ times in medium $S_{1}$ only. If $m_{1} \geq 1$ and $m_{2} \geq 1$, it means the wave propagates $m_{1}$ times in medium $S_{1}$ and $m_{2}$ times in medium $S_{2}$.

Consider the wave propagation process sketched in Figure 6; the reflected pressure wave on the wet surface of the panel with stiffener (T-shaped) can be summarized as

$$
\begin{aligned}
p_{\mathrm{ref}, m_{1}, m_{2}}^{(2)}\left(t_{m_{1}, m_{2}}, t\right) & = \begin{cases}r_{w s_{1}} \cdot p_{\text {in }}(t), & \text { for } m_{1}=m_{2}=0, \\
s_{w s_{1}} s_{s_{1} w}\left(r_{s_{1} w} r_{s_{1} s_{2}}\right)^{m_{1}-1} r_{s_{1} s_{2}} \cdot p_{\text {in }}\left(t-t_{m_{1}, 0}\right), & \text { for } m_{1} \geq 1, m_{2}=0, \\
\sum_{i=1}^{k} f^{i}\left(t_{m_{1}, m_{2}}, t\right), & \text { for } m_{1} \geq 1, m_{2} \geq 1,\end{cases} \\
p_{\text {ref }}^{(2)}(t) & =\sum_{i=0}^{m_{1}} \sum_{j=0}^{m_{2}} p_{\text {ref }, i, j}^{(2)}\left(t_{i, j}, t\right),
\end{aligned}
$$




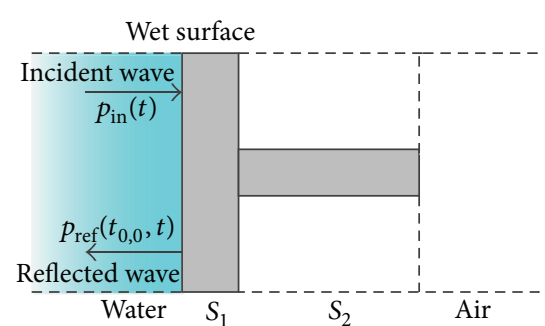

(a)

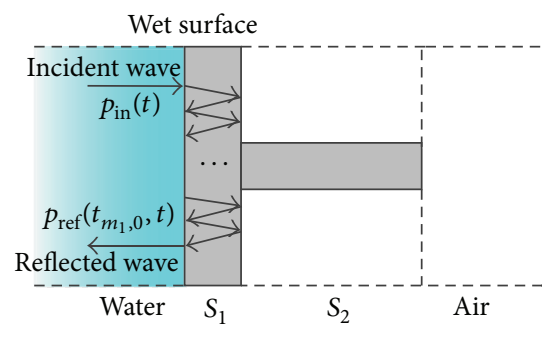

(b)

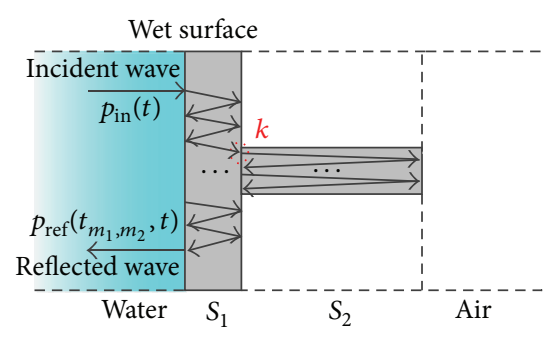

(c)

FIGURE 6: The wave propagation process in the panel with stiffener (T-shaped): (a) $m_{1}=0, m_{2}=0$; (b) $m_{1}>0, m_{2}=0$; (c) $m_{1}>0, m_{2}>0$.

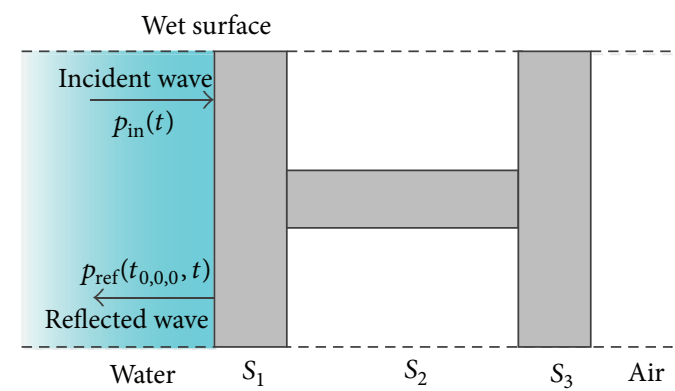

(a) $m_{1}=0, m_{2}=0$, and $m_{3}=0$

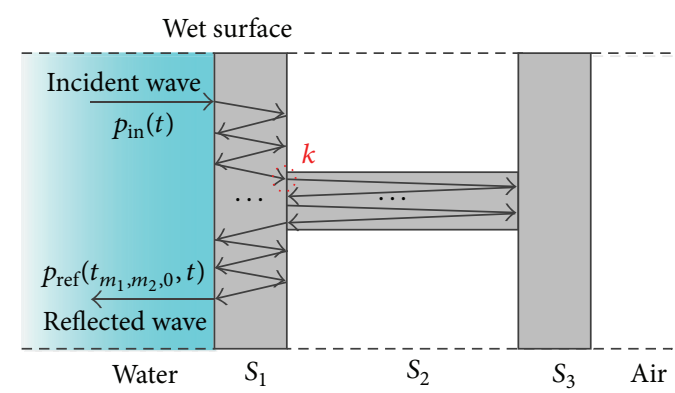

(c) $m_{1}>0, m_{2}>0$, and $m_{3}=0$

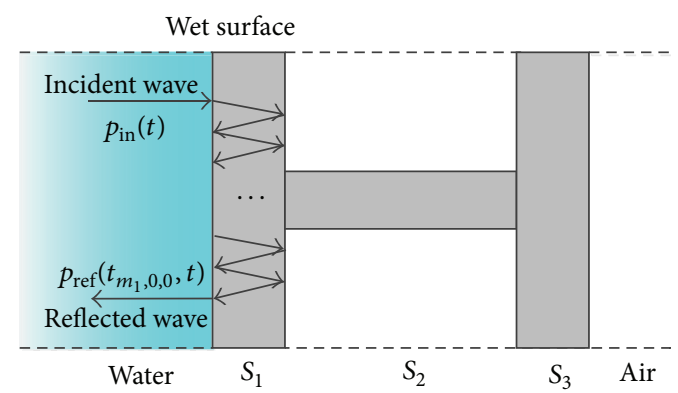

(b) $m_{1}>0, m_{2}=0$, and $m_{3}=0$

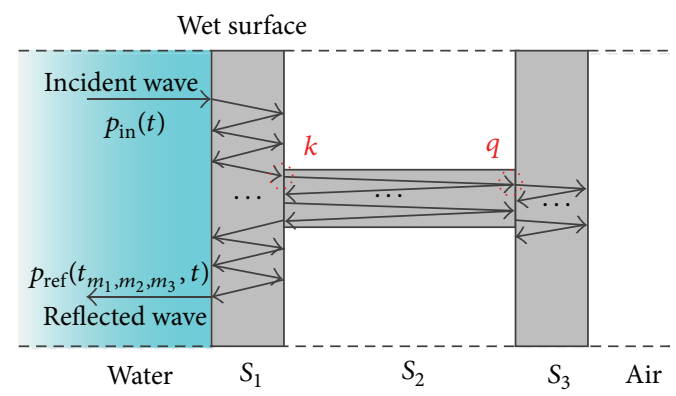

(d) $m_{1}>0, m_{2}>0$, and $m_{3}>0$

FIgURE 7: The different cases of the input wave propagates in the panel with girder (I-shaped).

where

$$
\begin{aligned}
& f^{k}(\left.t_{m_{1}, m_{2}}, t\right) \\
&=C_{m_{2}-1}^{k-1} C_{m_{1}}^{k} s_{w s_{1}} s_{s_{1} w}\left(s_{s_{1} s_{2}} r_{s_{2} a} s_{s_{2} s_{1}}\right)^{k}\left(r_{s_{1} w}\right)^{k-1} \\
& \cdot\left(r_{s_{1} w} r_{s_{1} s_{2}}\right)^{m_{1}-k}\left(r_{s_{2} a} r_{s_{2} s_{1}}\right)^{m_{2}-k} \cdot p_{\text {in }}\left(t-t_{m_{1}, m_{2}}\right), \\
& k=\min \left(m_{1}, m_{2}\right),
\end{aligned}
$$

where the superscript $k$ on $f^{k}\left(t_{m_{1}, m_{2}}, t\right)$ indicates $k$ times. If $k=1$, it means that the wave is transmitted once from medium $S_{1}$ to medium $S_{2}$ (see Figure 6(c)). The combinatorial number $C_{m_{1}}^{k}=m_{1} ! /\left[\left(m_{1}-k\right) ! k !\right]$ represents that from $m_{1}$ times of propagation occurances in mediums $S_{1}$ the wave transmits $k$ times into medium $S_{2} . C_{m_{2}-1}^{k-1}=\left(m_{2}-1\right) ! /\left\{\left[\left(m_{2}-\right.\right.\right.$ $1)-(k-1)] !(k-1) !\}$ means that the wave propagating $m_{2}$ times in medium $S_{2}$ is divided into $k$ parts. (c) Response of the Panel with Girder (I-Shaped). Consider the problem sketched in Figure 4(c); we assumed that the input wave propagates in media $S_{1}, S_{2}$, and $S_{3}$ for $m_{1}, m_{2}$, and $m_{3}$ times, respectively. The specific time $t_{m_{1}, m_{2}, m_{3}}$ can be written as

$$
t_{m_{1}, m_{2}, m_{3}}=m_{1} \cdot t_{2 S_{1}}+m_{2} \cdot t_{2 S_{2}}+m_{3} \cdot t_{2 S_{3}}
$$

where $m_{1}, m_{2}$, and $m_{3}$ can be taken from 0 to infinity. Let $t_{-}$total be the total calculation time; then, $m_{1}, m_{2}$, and $m_{3}$ mean the value of $t_{-}$total $/ t_{2 S_{1}}, t_{-}$total $/ t_{2 S_{2}}$, and $t_{-}$total $/ t_{2 S_{3}}$ rounded to the nearest integer, respectively.

We discuss this problem considering four scenarios (see Figure 7).

If $m_{1}=m_{2}=m_{3}=0$, it means the input wave does not transmit into medium $S_{1}$.

If $m_{1} \geq 1$ and $m_{2}=m_{3}=0$, as shown in Figure 7(b) the wave transmits from water into medium $S_{1}$ and propagates only in mediums $S_{1}$ for $m_{1}$ times. The wave does not transmit into medium $S_{2}$ or $S_{3}$. 
If $m_{1} \geq 1, m_{2} \geq 1$, and $m_{3}=0$, it indicates that the wave is not transmitted into mediums $S_{3}$. The wave transmission times from medium $S_{1}$ into medium $S_{2}$ can be from 1 to $k$. The number $k$ is the minimum of $m_{1}$ and $m_{2}$.

If $m_{1} \geq 1, m_{2} \geq 1$, and $m_{3} \geq 1$, it indicates the input wave propagates through the whole structure. The transmission times from medium $S_{2}$ into medium $S_{3}$ can be from 1 to $q$. The number $q$ is the minimum of $m_{1}, m_{2}$, and $m_{3}$.

To sum up, the reflected pressure on the wet surface of the panel with girder (I-shaped) at the specific time $t_{m_{1}, m_{2}, m_{3}}$ can be written as

$$
\begin{aligned}
p_{\text {ref }, i, j, k}^{(3)}\left(t_{m_{1}, m_{2}, m_{3}}, t\right) & = \begin{cases}r_{w s_{1}} \cdot p_{\text {in }}(t), & \text { for } m_{1}=m_{2}=m_{3}=0, \\
s_{w s_{1}} s_{s_{1} w}\left(r_{s_{1} w} r_{s_{1} s_{2}}\right)^{m_{1}-1} r_{s_{1} s_{2}} \cdot p_{\text {in }}\left(t-t_{m_{1}, 0,0}\right), & \text { for } m_{1} \geq 1, m_{2}=m_{3}=0, \\
\sum_{i=1}^{k} f^{k}\left(t_{m_{1}, m_{2}, 0}, t\right), k=\min \left(m_{1}, m_{2}\right), & \text { for } m_{1} \geq 1, m_{2} \geq 1, m_{3}=0, \\
\sum_{i=1}^{q} \sum_{i=1}^{k} f^{k q}\left(t_{m_{1}, m_{2}, m_{3}}, t\right), q=\min \left(m_{1}, m_{2}, m_{3}\right), & \text { for } m_{1} \geq 1, m_{2} \geq 1, m_{3} \geq 1,\end{cases} \\
p_{\text {ref }}^{(3)}(t) & =\sum_{i=0}^{m_{1}} \sum_{j=0}^{m_{2}} \sum_{k=0}^{m_{3}} p_{\text {ref }, i, j, k}^{(3)}\left(t_{i, j, k}, t\right),
\end{aligned}
$$

where

$$
\begin{aligned}
f^{k} & \left(t_{m_{1}, m_{2}, 0}, t\right)=C_{m_{2}-1}^{k-1} C_{m_{1}}^{k} s_{w_{s_{1}}} s_{s_{1} w}\left(s_{s_{1} s_{2}} r_{s_{2} s_{3}} s_{s_{2} s_{1}}\right)^{k} \\
\cdot & \left(r_{s_{1} w}\right)^{k-1}\left(r_{s_{1} w} r_{s_{1} s_{2}}\right)^{m_{1}-k}\left(r_{s_{2} s_{3}} r_{s_{2} s_{1}}\right)^{m_{2}-k} \\
\cdot & p_{\text {in }}\left(t-t_{m_{1}, m_{2}, 0}\right), \\
f^{k q} & \left(t_{m_{1}, m_{2}, m_{3}}, t\right) \\
= & C_{m_{3}-1}^{q-1} C_{m_{2}}^{q} C_{m_{2}-1}^{k-1} C_{m_{1}}^{k} s_{w s_{1}} s_{s_{1} w}\left(s_{s_{1} s_{2}} s_{s_{2} s_{1}}\right)^{k} \\
\cdot & \left(r_{s_{1} w}\right)^{m_{1}-1}\left(r_{s_{1} s_{2}}\right)^{m_{1}-k}\left(r_{s_{2} s_{1}}\right)^{m_{2}-k} \cdot\left(s_{s_{3} s_{2}} s_{s_{2} s_{3}}\right)^{q} \\
\cdot & \left(r_{s_{2} s_{3}}\right)^{m_{2}-q}\left(r_{s_{3} s_{2}}\right)^{m_{3}-q} r_{s_{3} a}^{m_{3}} \cdot p_{\text {in }}\left(t-t_{m_{1}, m_{2}, m_{3}}\right) .
\end{aligned}
$$

The pressure of the wave that transmits $k$ times from $S_{1}$ into medium $S_{2}$ and transmits $q$ times from $S_{2}$ into medium $S_{3}$ is denoted as $f^{k q}\left(t_{m_{1}, m_{2}, m_{3}}, t\right)$. The combinatorial number $C_{m_{2}}^{q}$ indicates that from $m_{2}$ times of propagation occurrences the wave is transmitted $q$ times from $S_{2}$ into medium $S_{3}$. $C_{m_{3}-1}^{q-1}$ implies that the wave propagating in medium $S_{3}$ is divided into $q$ parts.

As listed above, the plate velocity and pressure for three substructures prior to the onset of first cavitation can be summarized as

$$
\begin{aligned}
& p_{\text {wet }}^{(k)}(t)=p_{\text {in }}(t)+p_{\text {ref }}^{(k)}(t), \quad k=1,2,3, \\
& v_{\text {wet }}^{(k)}(t)=\frac{p_{\text {in }}(t)}{\rho_{w} c_{w}}-\frac{p_{\text {ref }}^{(k)}(t)}{\rho_{w} c_{w}}, \quad k=1,2,3,
\end{aligned}
$$

where the superscript $(k)$ represents the simple panel, the panel with stiffener (T-shaped), and the panel with girder (I-shaped), respectively. $v_{\text {wet }}^{(k)}(t)$ is the velocity of the fluidstructure interface.
2.2.2. Response of Three Substructures Subsequent to the First Cavitation. In this section, the analysis is performed with the assumption that the water response can be described by a linear-elastic, reversible pressure versus volumetric strain constitutive relation for $p>0$. In contrast, we assume that the fluid is unable to sustain any pressure for $p<0$; then, we obtain

$$
p= \begin{cases}-\rho_{w} c_{w}^{2} \varepsilon_{V}, & \text { for } p>0, \\ 0, & \text { for } p \leq 0 .\end{cases}
$$

The pressure in the water between the reflected wave front and the wet face is

$$
\begin{aligned}
& p_{w}^{(k)}(x, t)=p_{\text {in }}\left(t-\frac{x}{c_{w}}\right)+p_{\text {ref }}^{(k)}\left(t+\frac{x}{c_{w}}\right), \\
& k=1,2,3 .
\end{aligned}
$$

For $-t c_{w} \leq x \leq 0$, the pressure given by (16) is valid until the water first cavities. Prior to cavitation, the velocity in the fluid between the reflected wave front and wet surface is presented as

$$
v_{w}^{(k)}(t)=\frac{p_{\text {in }}\left(t-x / c_{w}\right)}{\rho_{w} c_{w}}-\frac{p_{\mathrm{ref}}^{(k)}\left(t+x / c_{w}\right)}{\rho_{w} c_{w}}, \quad \begin{aligned}
& \\
& k=1,2,3,
\end{aligned}
$$

where the superscript $(k)$ represents the simple panel, the panel with stiffener (T-shaped), and the panel with girder (Ishaped), respectively.

The cavitation time $t_{\mathrm{cav}}(x)$ at a location $x$ in the water can be obtained by setting the pressure to zero in (16) and solving the implicit Eq. $p_{w}^{(k)}\left(x, t_{x}\right)=0$. By taking the minimum value of $t_{\text {cav }}(x)$, we can obtain the location and time of first cavitation, which are denoted by $x_{c}$ and $t_{c}$. According 
to the location and time of first cavitation, we can achieve the pressure and velocity response subsequent to the first cavitation for three cases.

Case 1. If $t_{c}=t_{\text {cav }}(0)$ or $x_{c}=0$, it means the first cavitation takes place on the wet surface. A new breaking front forms. In this case, we assume that the pressure on the wet surface is zero and the velocity is given as an average. Therefore, for $t \geq t_{c}$ the pressure and velocity are presented as

$$
\begin{aligned}
& p_{\text {wet }}^{(k)}(t)=0, \\
& v_{\text {wet }}^{(k)}(t)=\frac{\int_{0}^{t_{c}^{(k)}} p_{\text {wet }}^{(k)}(t) d t}{\rho_{s}^{(k)} H_{\mathrm{eq}}^{(k)}},
\end{aligned}
$$

where $H_{\mathrm{eq}}^{(k)}$ refer to the corresponding solid plate thickness.

Case 2. If $t_{c}<t_{\text {cav }}(0)$ or $x_{c}<0$ and $x_{c}<x_{\text {rest }}<0, t_{c}<t_{\text {rest }}$, it means after the first cavitation $t>t_{c}$ two breaking waves propagate towards opposite directions at a speed in excess of sound speed $c_{w}$. The breaking front traveling towards the wet surface can be arrested at position $x_{\text {rest }}$ and time $t_{\text {rest }}$ before reaching the fluid-structure interface (see Figure 8).

The conditions for propagation of a breaking front in the water are given by Kennard [16] as

$$
\begin{aligned}
&\left.p_{w}\right|_{x=x_{\mathrm{bf}}}=0 ; \\
&\left.\frac{\partial v_{w}}{\partial x}\right|_{x=x_{\mathrm{bf}}}>0 ; \\
&\left.\left|\frac{\partial p}{\partial x}\right|_{x=x_{\mathrm{bf}}}\right|_{\left.\frac{\partial p}{\partial x}\right|_{x=x_{\mathrm{bf}}} \geq}<\left.\rho_{w} c_{w} \frac{\partial v_{w}}{\partial x}\right|_{x=x_{\mathrm{bf}}}, \\
& \quad \text { (propagation in the }+x \text { direction) }, \\
&\left.\frac{\partial p}{\partial x}\right|_{x=x_{\mathrm{bf}}} \leq 0 \\
& \quad \text { (propagation in the }-x \text { direction) },
\end{aligned}
$$

where $x_{\mathrm{bf}}$ is the current position of the breaking front. Once the breaking front traveling in the $+x$ direction has been arrested, if at least one of the conditions fails, a closing front can take place and propagate in the $-x$ direction. Consequently, propagation of this front begins at position $x_{\text {rest }}$ and time $t_{\text {rest }}$. The pressure and particle velocities at the closing front denoted by $p_{\mathrm{cf}}$ and $v_{\mathrm{cf}}$ are given by

$$
\begin{aligned}
& p_{\mathrm{cf}}=p_{\mathrm{cf},-}+p_{\mathrm{cf},+}, \\
& v_{\mathrm{cf}}=v_{\mathrm{cf},-}+v_{\mathrm{cf},+}=\frac{-p_{\mathrm{cf},-}+p_{\mathrm{cf},+}}{\rho_{w} c_{w}},
\end{aligned}
$$

where $p_{\mathrm{cf},-}$ and $p_{\mathrm{cf},+}$ are wave trains approaching the cavitation region and traveling away from the cavitation region, respectively. $v_{\mathrm{cf},-}$ and $v_{\mathrm{cf},+}$ are particle velocities associated with the wave trains $p_{\mathrm{cf},-}$ and $p_{\mathrm{cf},+}$, respectively. According to Kennard, we can have the conditions for propagation of a closing front

$$
\begin{gathered}
2 p_{\mathrm{cf},-}>-\rho_{w} c_{w} v_{\mathrm{cav}}(x) ; \\
\eta(x, t)=\int_{t_{\mathrm{bf}}(x)}^{t} \frac{\partial v_{\mathrm{cav}}(x)}{\partial x} d t,
\end{gathered}
$$

where $v_{\text {cav }}(x)$ represents the particle velocity of the cavitated water, evaluated at a position just ahead of the closing front, namely, $v_{\text {cav }}\left(x_{\mathrm{bf}}\right)=v_{w}\left(x_{\mathrm{bf}}, t_{\mathrm{bf}}\right)$. The quantity $\eta(x, t)$ represents a measure of the strain in the cavitation water. The momentum and mass conservation at the closing front in the original coordinates (see Figure 8) are

$$
\begin{array}{r}
p_{\mathrm{cf}}=(1-\eta) \rho_{w} \frac{d x_{\mathrm{cf}}}{d t}\left(v_{\mathrm{cf}}-v_{\mathrm{cav}}\right), \\
\rho_{w} c_{w}^{2}\left(v_{\mathrm{cf}}-v_{\mathrm{cav}}\right)=\left[\rho_{w} c_{w}^{2} \eta+p_{\mathrm{cf}}(1-\eta)\right] \frac{d x_{\mathrm{cf}}}{d t} .
\end{array}
$$

Equations (20) and (22) can be rewritten as

$$
\begin{aligned}
\frac{d x_{\mathrm{cf}}}{d t} & =-\frac{c_{w} \lambda}{\lambda+\left(c_{w}-\lambda\right) \eta}, \\
p_{\mathrm{cf}} & =\frac{(1-\eta) \rho_{w} c_{w} \lambda^{2}}{2 \lambda+\left(c_{w}-2 \lambda\right) \eta},
\end{aligned}
$$

where $\lambda$ is the auxiliary quantity and is expressed as $\lambda=$ $2 p_{\mathrm{cf},-} / \rho_{w} c_{w}+v_{\mathrm{cav}}$.

According to Figure 8, the solution in Case 2 can be calculated by three steps.

In the first step, $t=t_{\text {rest }}$, the closing front pressure $p_{\mathrm{cf}}$ is given by the expression prior to the onset of first cavitation. $p_{\mathrm{cf},-}$ and $p_{\mathrm{cf},+}$ can be written as

$$
\begin{aligned}
& p_{\mathrm{cf},-}=p_{\text {ref }}\left(t+\frac{x}{c_{w}}\right), \\
& p_{\mathrm{cf},+}=p_{\text {in }}\left(t-\frac{x}{c_{w}}\right) .
\end{aligned}
$$

In the second step, $t_{\text {rest }}<t<t_{\text {rest }}-x_{\text {rest }} / c_{w}$, the $p_{\text {cf,- }}$ is given by (24). By solving (23) and checking if the conditions in (19) are satisfied, we can obtain wave trains traveling away from the cavitation region $p_{\mathrm{cf},+}$ and update the current position of closing front $x_{\mathrm{cf}}$. The value of $p_{\mathrm{cf},+}$ is used to generate a wave train which will act on the plate at $t_{\mathrm{cf}}-x_{\mathrm{cf}} / c_{w}$.

In the third step, $t \geq t_{\text {rest }}-x_{\text {rest }} / c_{w}$, the wave trains $p_{\mathrm{cf},+}$ reaches the wet surface invalidating the expression prior to the onset of first cavitation for the plates velocity. The analysis of the closing front propagation is similar to the process during $t_{\text {rest }}<t<t_{\text {rest }}-x_{\text {rest }} / c_{w}$ in the second step. $p_{\text {in }}(t)$ is given by

$$
p_{\text {in }}(t)=p_{\text {cf, },}\left(t-t_{\text {rest }}+\frac{x_{\text {rest }}}{c_{w}}\right) .
$$

Based on (25), we can compute the plate pressure and velocity expression subsequent to the first cavitation by solving (13) and (14). 

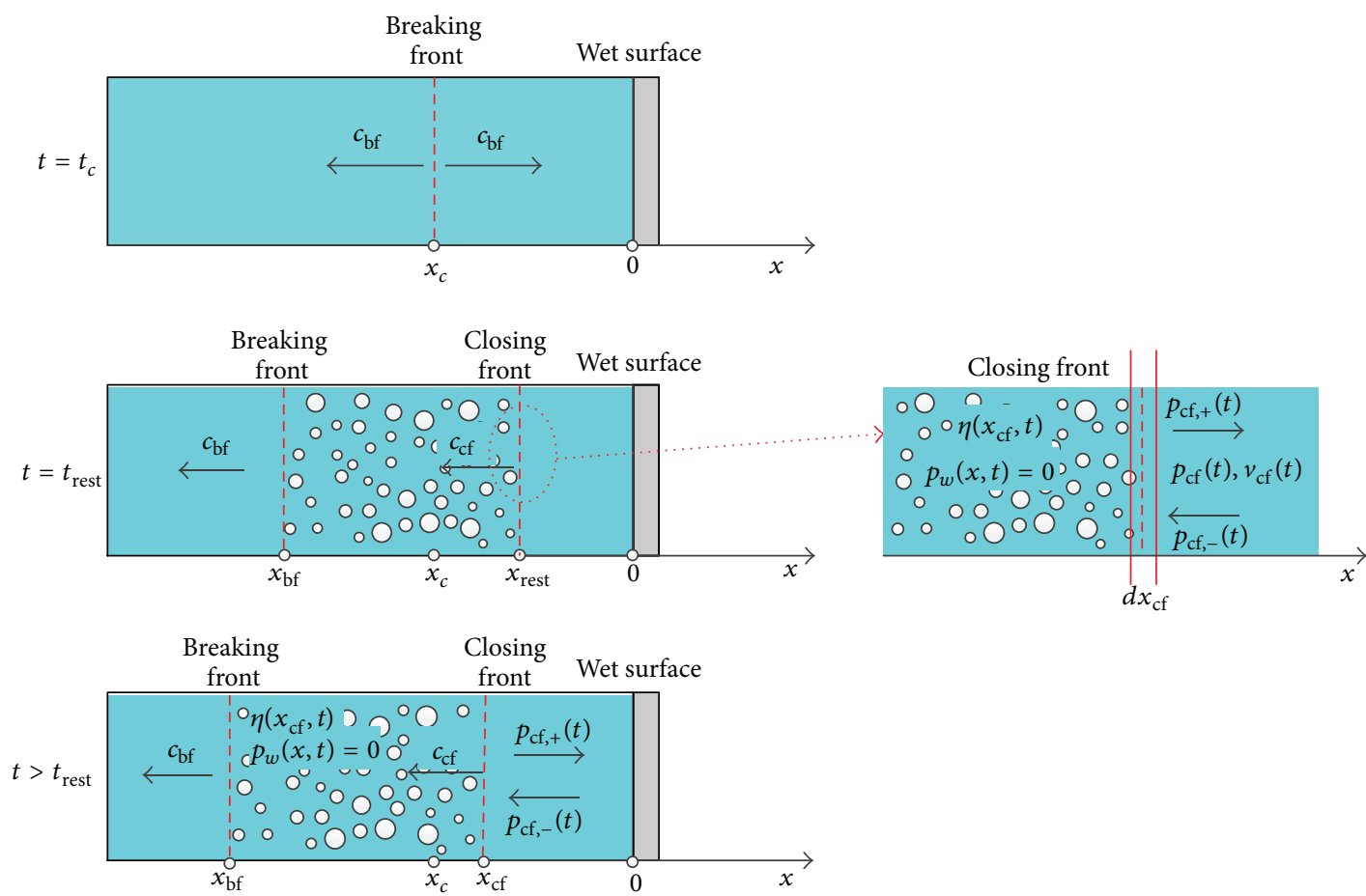

FIGURE 8: Schematic illustration of the phenomena of initial cavitation, emergence and propagation of breaking fronts, and development of a closing front.

\section{Validation}

3.1. Finite Element Model. As shown in Figure 9, the threedimensional numerical model consists of a water column on the left side of the wet surface. Contact is enforced at the fluidstructure interface, and the free-standing plate is modeled by applying symmetry boundary conditions. The commercial code ABAQUS [31] is used to validate the analytical models described in Section 2.2.

Three-dimensional FE simulations were performed to provide more insight into fluid and structural response. For the corresponding substructures, we consider a homogeneous material with $\rho=7800 \mathrm{Kg} / \mathrm{m}^{3}$, Young's modulus $E=2.11 e 11 \mathrm{~Pa}$, and Poisson's ratio $\mu=0.3$. The dimensions of the substructures are shown in Table 1. The eight-node three-dimensional brick elements with reduced integration (C3D8R) are used to model the structure. The element meshes of substructures are shown in Figure 10. To capture the stress wave in the structure, the element sizes $l_{e s 1_{l},}, l_{e s 2 \_l}$, and $l_{e s 3 l}$ along the longitudinal direction of the substructures are set to $0.1 \mathrm{~mm}$. The element sizes $l_{e s 1 \_t}, l_{e s 2 . t}$, and $l_{e s 3 \_t}$ of $10 \mathrm{~mm}$ are used to discretize the substructures along the width, while a single element is used to discretize the substructures along the depth. The elements of the panel, the panel with stiffener (T-shaped), and the panel with girder (Ishaped) are 700, 1400, and 9400, respectively.

In ABAQUS, by using a Mie-Gruneisen equation of state with a linear Hugoniot relation, we can model the water with the linear-elastic relation described in (15). The density and sound speed of water are set to $\rho_{w}=1000 \mathrm{Kg} / \mathrm{m}^{3}$ and $c_{w}=$ $1500 \mathrm{~m} / \mathrm{s}$, respectively. The bulk modulus of water is $K_{w}=$

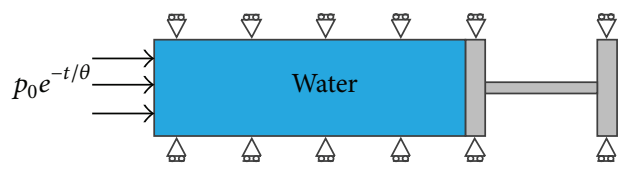

FIGURE 9: A sketch of the boundary conditions in the analysis of free-standing panel with girder (I-shaped) impinged by a water blast.

$\rho_{w} c_{w}^{2}=2.25 \mathrm{GPa}$. The shear modulus and hydrostatic tensile strength of water are set to be zero. Acoustic eight-node three-dimensional brick elements with reduced integration (AC3D8R) are employed to model the water column. To capture the cavitation effect in the water column, the length of the water column is set to $1.2 \mathrm{~m}$. The element size $l_{e w \perp}$ of the water along the longitudinal direction of the water column is set to $0.3 \mathrm{~mm}$, while the element size $l_{\text {ew_t }}$ of $10 \mathrm{~mm}$ is used to discretize the water along the width, while one single element is used to discretize the water along the depth. The water column comprises 28000 elements.

The water cavitation effects are realized by total wave formulation. We set the cavitation limit to be zero and the hydrostatic pressure field in the water column to be sufficiently small. A uniform pressure is imposed on the left surface of the water column. The impulses used all have peak pressure $p_{0}=10 \mathrm{MPa}$ and the decay constant $\theta=0.1 \mathrm{~ms}$.

3.2. Comparison of Analytical and FE Prediction. In this section, we compare the analytical and FE predictions for fluid-structure interface pressure and velocity histories, as 


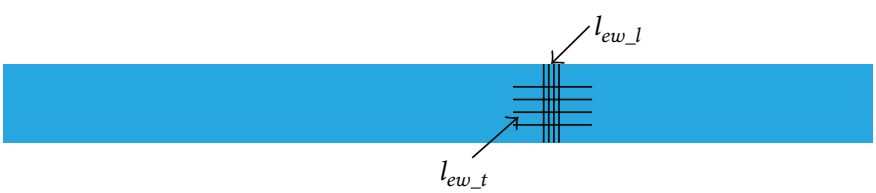

(a)

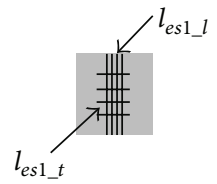

(b)

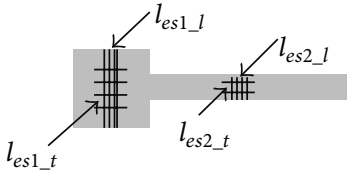

(c)

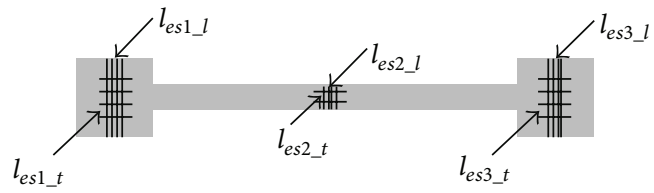

(d)

FIGURE 10: The mesh of fluid and structure: (a) the water column; (b) the panel with stiffener (T-shaped); (c) the panel with girder (I-shaped).

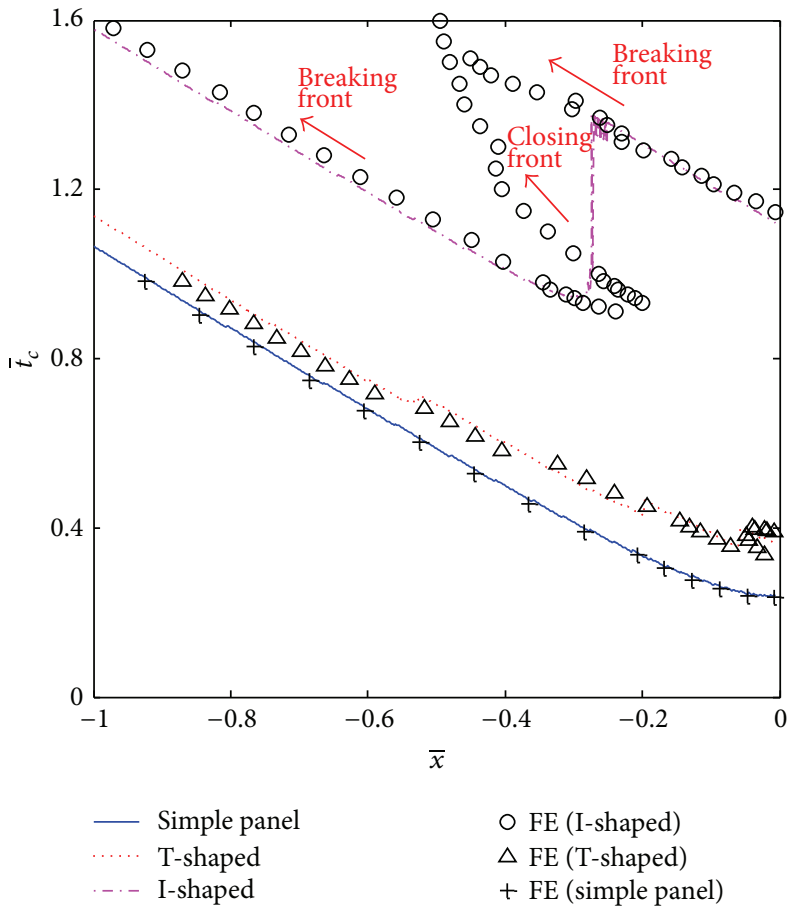

(a)

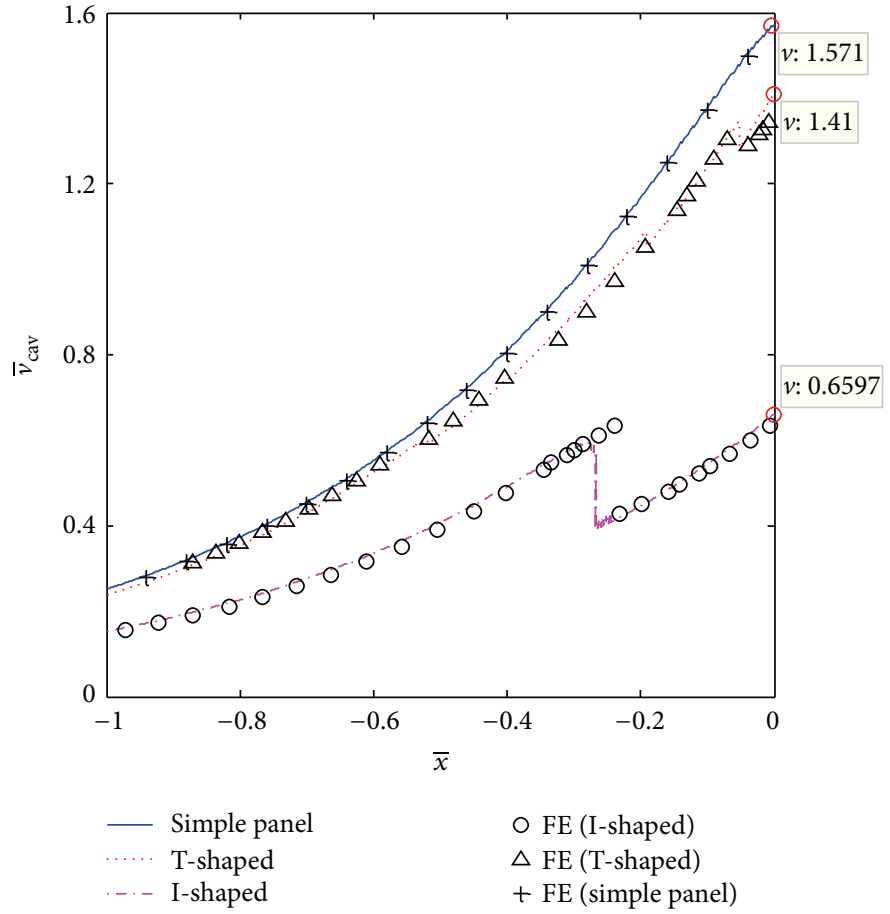

(b)

FIGURE 11: Analytical predictions and FE prediction of the spatial variation of (a) the time to cavitation and (b) the velocity $\bar{v}_{\text {cav }}$ in the cavitated water at $\bar{t}_{c}(x)$. These results are for cases in Table 1, which are the simple panel, the panel with stiffener (T-shaped), and the panel with girder (I-shaped).

well as the motion of breaking and closing fronts, to assess the accuracy of the analytical models.

For simplicity, we define nondimensional parameters: $\bar{t}=$ $t / \theta, \bar{x}=x /\left(c_{w} \theta\right), \bar{p}=p / p_{0}, \bar{v}=p /\left[p_{0} /\left(\rho_{w} c_{w}\right)\right]$, and $\bar{I}=$ $I /\left(p_{0} \theta\right)$, where $\bar{t}, \bar{x}, \bar{p}, \bar{v}$, and $\bar{I}$ are the nondimensional time, displacement, pressure, velocity, and impulse, respectively.

3.2.1. Closing Front and Breaking Front. Figure 11 presents the cavitation time $\bar{t}_{c}$ as a function of the location $\bar{x}$ and the velocity $\bar{v}_{\text {cav }}$ in the cavitated water at $\bar{t}_{c}(x)$ for the three substructures. The minimum value on these curves (circled in Figure 11(a)) is the location and time of the onset of first cavitation. Two branches of each curve originated from this point, corresponding to propagation of two breaking fronts traveling away from (left of $\bar{x}_{c}$ ) and towards (right of $\bar{x}_{c}$ ) the wet surface. Note that the cavitation time is linear with $\bar{x}$ on the left of $\bar{x}_{c}$, except very close to the first cavitation location.

For the simple panel, cavitation initiates at a very small distance from the wet surface and gives rise to a breaking front traveling away from the interface, while the breaking front approaching the interface cannot be arrested before reaching the wet surface. For the panel with stiffener ( $\mathrm{T}$ shaped) and the panel with girder (I-shaped), the first cavitation is located within the fluid and the breaking front traveling towards the structure would reverse its motion and become the closing front. Figure 11(a) shows the first 
cavitation time for the water column. For example, the jump in the curve of the panel with girder (I-shaped) indicates that when the first cavitation occurs at $\bar{x}=-0.298$, the breaking front propagated away from the wet surface. As time elapses, another cavitation takes place at the interface at time $\bar{t}_{c}(0)$. And this breaking front also propagated away from the wet surface. These two breaking fronts generate from different place, while they travel at the same velocity. The jump means that the cavitation will occur twice at the same displacement and different time. We can see a similar phenomenon in the case of the panel with stiffener (T-shaped).

As shown in Figure 11(a), the trends of two breaking fronts in the analytical results agree well with FE results. The analytical results in Figure 11(a) only show the first cavitation time for the water column. Thus, when the cavitation occurs at the same displacement and different time, the curve will "jump" without showing the closing front. And this leads to the discrepancy between FE and analytical predictions. From Figure 11(a), we can see that $\bar{t}_{c}(0)<t_{\text {rest }} / \theta-x_{\text {rest }} /\left(c_{w} \theta\right)$ indicates that the cavitation occurs on the wet surface before the wave train emanating from the closing front reaches the plate.

The expressions (13) for the plate velocity and pressure are still valid when $\bar{t}<\bar{t}_{c}(0)$. In Figure 11(b), the velocity $\bar{v}_{\text {cav }}$ in the cavitated water at $\bar{t}_{c}(x)$ for the three substructures is shown and small difference is found between the analytical solutions and the FE predictions. The particle velocity $\bar{v}_{\text {cav }}(0)$ is the corresponding velocity on the wet surface when cavitation occurs. The value of $\bar{v}_{\text {cav }}(0)$ for the panel, the panel with the stiffener (T-shaped), and the panel with girder (Ishaped) are $1.568,1.407$, and 0.6657 , respectively. The variance from the analytical results for the different shape plates is large. This is due to the fact that the panel with girder (Ishaped) has a larger unit mass.

3.2.2. Pressure and Velocity Histories on Wet Surface. Figure 12(a) shows nondimensional time histories of nondimensional wet surface pressure $\bar{p}_{\text {wet }}^{(1)}$, while the corresponding time histories of nondimensional velocity $\bar{v}_{\text {wet }}^{(1)}$ are presented in Figure 12(b). As discussed above, in this particular case the first cavitation takes place at a location very close to the wet surface. We can then calculate the analytical solution of the pressure and velocity by using (18) after the wet surface is cavitated. In particular, we investigated the dynamic response of a steel plate by a three-method analytical solution, FEM results, and Taylor's solution and then did a comparison of the predictions. Both Figures 12(a) and 12(b) show that the analytical solutions are adequately predicted by $\mathrm{FE}$ results and Taylor's solutions [8]. On the whole process, the analytical solution agrees well with the FE prediction for the pressure and velocity. It is to be noted that the small jumps of analytical solutions indicate the reflections and transmissions of the pressure wave. When the stress wave propagation and the cavitation effect are considered, the analytical results are more accurate than Taylor's results and FE predictions.

To reveal the fluid-structure interaction of the doublebottom structure, the nondimensional time histories of wet surface pressure and velocity for the panel with stiffener (Tshaped) and the panel with girder (I-shaped) are shown in Figures 13 and 14, respectively. The FE predictions are presented to validate the analytical solutions. As shown, the analytical solutions agree considerably well with the FE predictions. The oscillations in FE predictions can be explained by the fact that on one hand the FE algorithm for the thin steel plate accounts for the FE predictions that have large numerical diffusions; on the other hand, the FE numerical models are three-dimensional, including the effect of the transverse wave. We note that if cavitation occurs on the wet surface, the velocity of interface would be given as an average. After the wet surface gets cavitated, the process becomes very complex to solve analytically. Thus, the velocity is calculated as an average. Hence, we can conclude that the analytical solutions are validated in these cases.

Note that the obvious drops in the analytical solutions indicate the superposition of the reflected wave from the rear surface of the structure and the incident wave. For the panel with stiffener (T-shaped), this reflected wave is the rarefaction wave which causes the great drop in the pressure and the decrement of decay constant (see Figure 13(a)). The specific time ticks circled in Figure 13(a), $t=0.05, t=0.105$, and $t=0.1583$, are the time ticks of stress wave traveling once, twice, and thrice through the panel with stiffener (T-shaped), namely, the time it takes for the reflected wave from the rear surface of the structure to reach the wet surface. Between two arbitrary time ticks, small oscillations exist. This is as a result of the superposition of the incident wave and the reflected wave from the interface of medium $S_{1}$ and medium $S_{2}$. As such, we can characterize the response of the panel with stiffener (T-shaped) by the drops in specific time tick and the small oscillations between the time ticks. The response clearly shows the wave propagation process in T-shaped plate. In Figure 13(b), the characteristics induced by superposition of multiwaves can be expressed as the jumps at specific time ticks and small oscillations between time ticks. Circles in Figure 13(b) are the maximum value of the velocity and the velocity when the wet surface gets cavitated, that is, 1.422 and 1.404. The panel with stiffener (T-shaped) gets its peak velocity before cavitation occurs on the wet surface. As shown, the analytical solution is a little larger than the FE prediction and the differences are reasonably acceptable. As shown in Figure 13, Taylor's solutions for rigid plate equivalent to panel with stiffener (T-shaped) have similar trend as the analytical and FE prediction. However, Taylor's solution cannot show the stress wave propagation process in the specific structure and it cannot recognize the minor change in the structure.

In Figure 14(a), we can see the obvious small jumps at the specific time ( $t=0.53, t=0.1068)$ which is the result of the enlargement effect when the stress wave is transmitted from medium $S_{2}$ to medium $S_{3}$. The details of the enhancement of pressure will be expressed in Section 4.2. The sharp drop of pressure occurs when the rarefaction wave from the rear surface of the structure reaches the wet surface. Similar to the wave propagation process in the panel with stiffener (Tshaped), the corresponding response of the panel with girder (I-shaped) is characterized by big drops at specific time tick and small oscillations between time ticks. The differences 


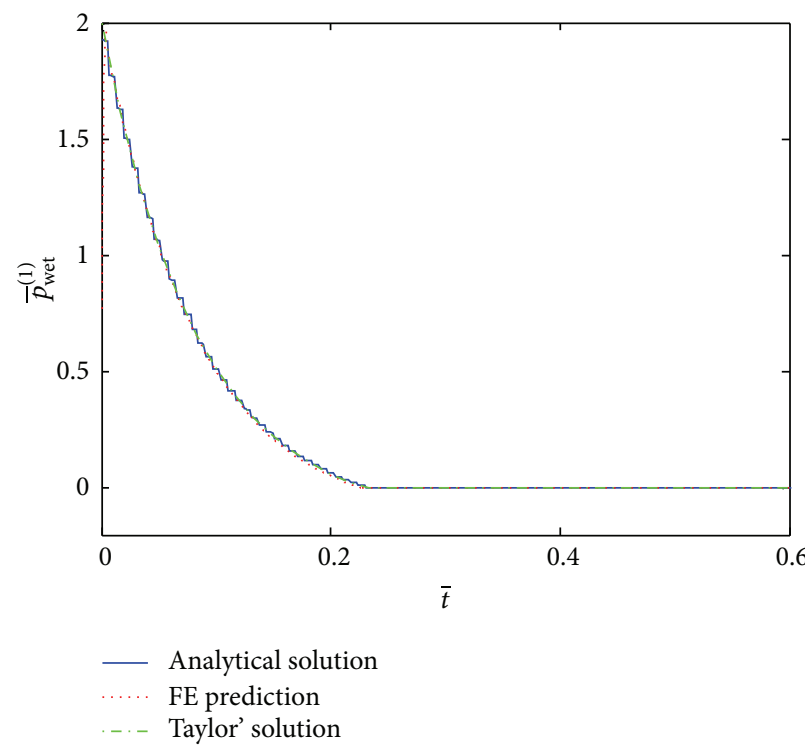

(a)

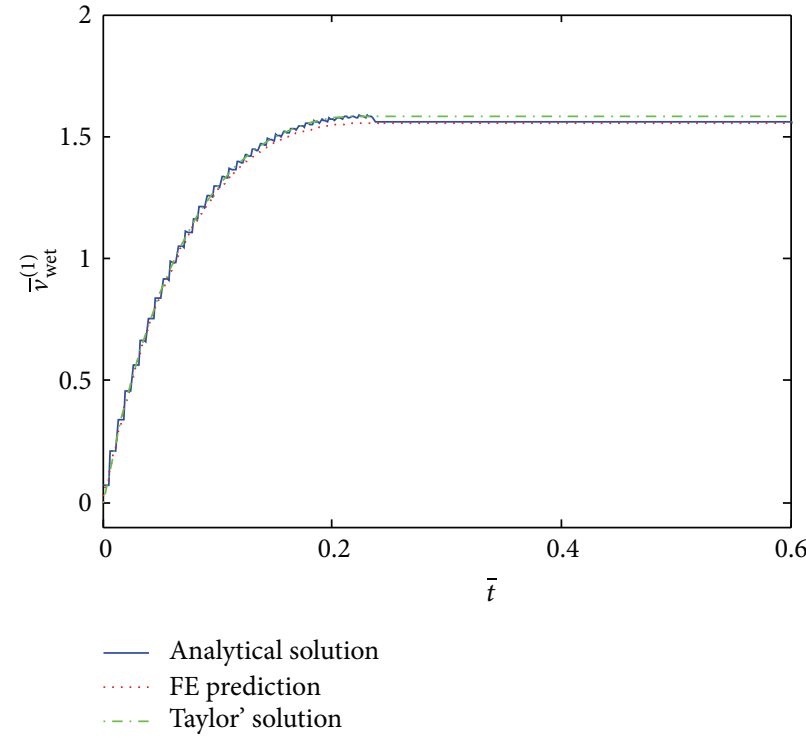

(b)

FIGURE 12: Nondimensional time histories of (a) nondimensional wet surface pressure and (b) nondimensional velocity for the simple panel.

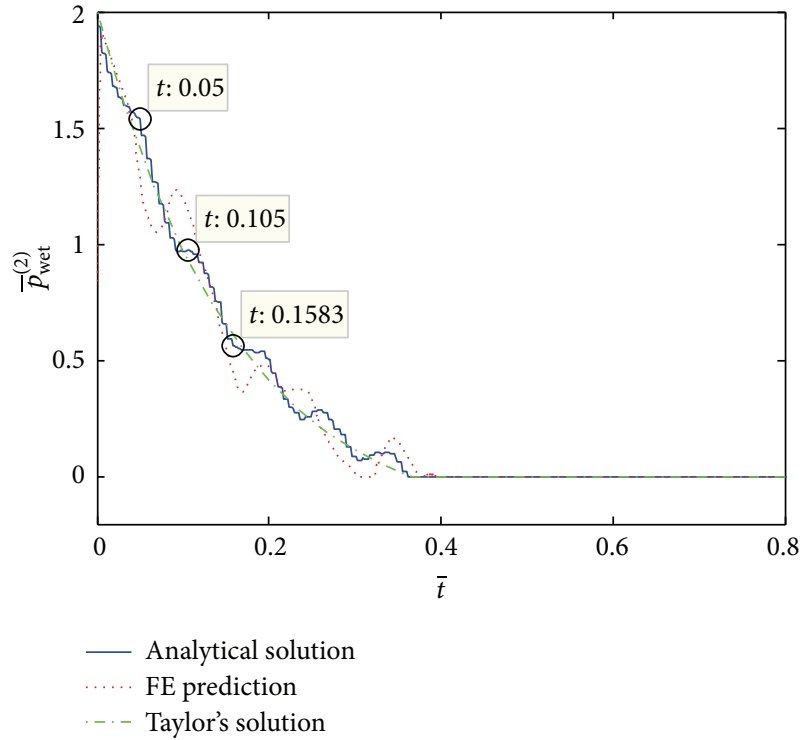

(a)

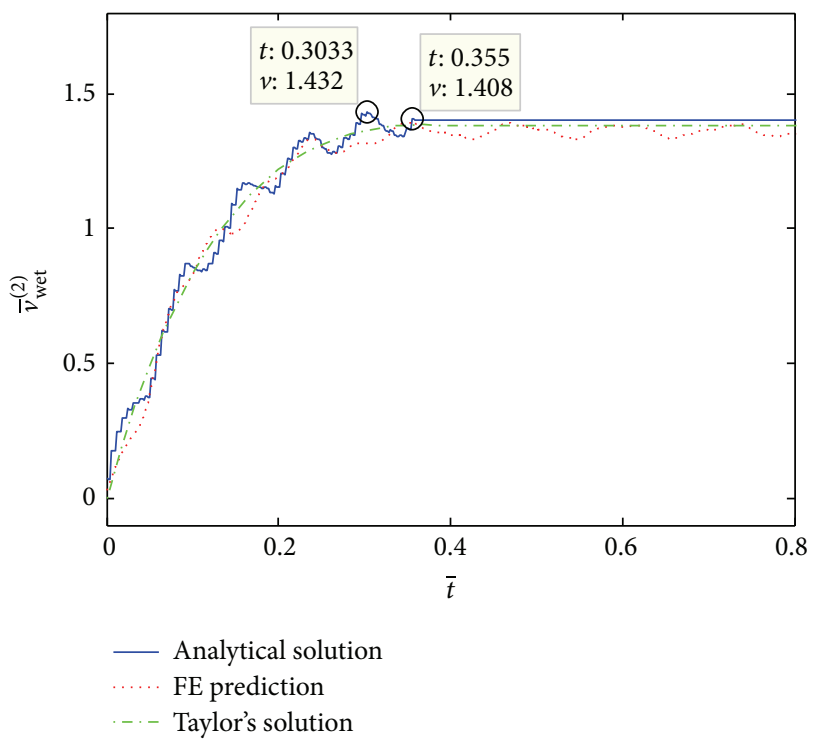

(b)

FIGURE 13: Nondimensional time histories of (a) nondimensional wet surface pressure and (b) nondimensional velocity for the panel with stiffener (T-shaped).

are that the wave travel in the panel with girder (I-shaped) takes longer time to finish one trip through the structure and the small jumps take place due to the existence of the medium $S_{3}$. In Figure 14(b), we also can describe the trends in nondimensional time history of velocity as jumps at specific time ticks and small oscillations between time ticks, as well as the small drops owing to the existence of the medium $S_{3}$. The panel with girder (I-shaped) gets its peak velocity before cavitation occurs on the wet surface (see circles in
Figure 14(b)). By comparing the velocities in Figures 12(b), 13(b), and 14(b), we can conclude that the velocity of the wet surface is considerably small if the structure is heavy or strong. Correspondingly, the rarefaction wave radiated by the motion of wet surface is small. Thus, the pressure on the wet surface decreases slowly (see Figure 14(a)). As shown in Figure 14, Taylor's solutions for rigid plate equal to the panel with girder (I-shaped) have a similar trend as the analytical and FE predictions. However, there is a big difference between 


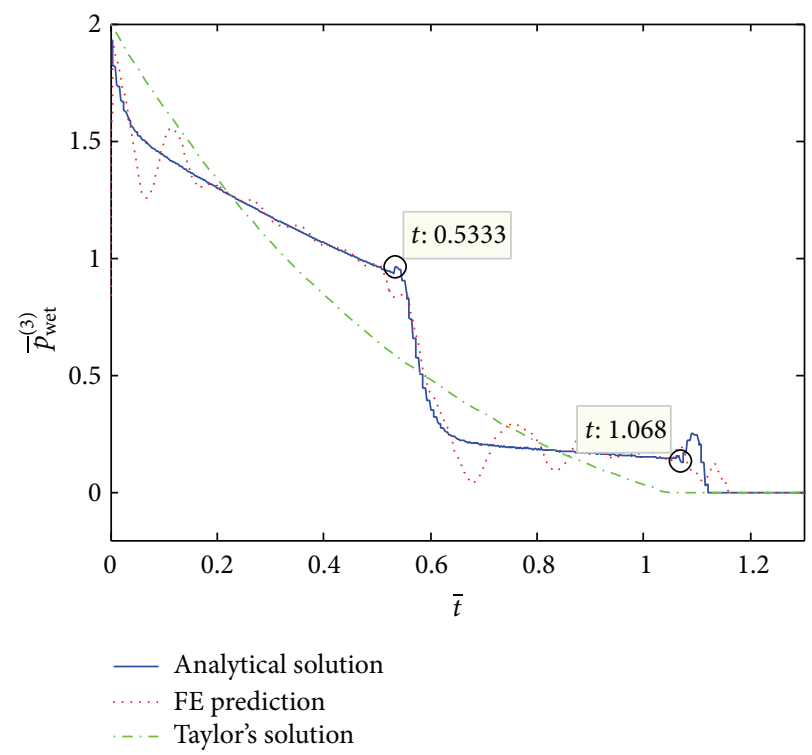

(a)

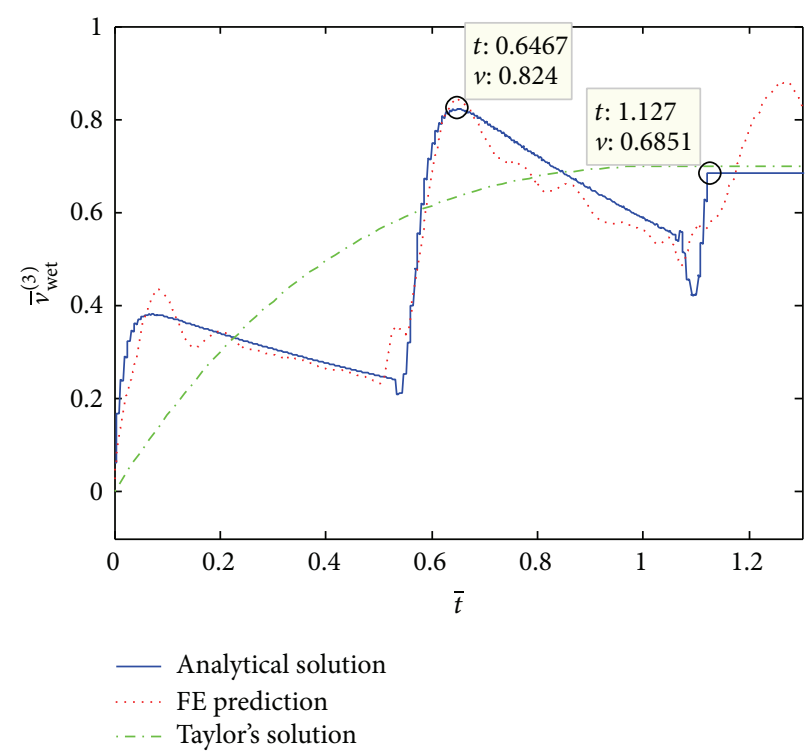

(b)

FIGURE 14: Nondimensional time histories of (a) nondimensional wet surface pressure and (b) nondimensional velocity for the panel with girder (I-shaped).

Taylor's solution and FE predictions. It can be seen that Taylor's solution cannot capture the reflected wave from the varying cross section of the structure.

3.2.3. Impulse Histories on Wet Surface. The intensity of loads impacted on the structure consequent to underwater explosion, as quantified by the specific impulse (per unit area) applied on the wet surface, is deduced by solving the following equation:

$$
I^{(k)}(t)=\int_{0}^{t}\left[p_{\text {wet }}^{(k)}(\tau)\right] d \tau, \quad k=1,2,3
$$

where $k=1,2,3$ refers to the simple panel, the panel with stiffener (T-shaped), and the panel with girder (I-shaped), respectively.

Define the maximum incident impulse $I_{0}$ (integral of the incident wave) per unit quasistatic state as $I_{0}=2 p_{0} \theta$. The severity of structural loading consequent to underwater explosion can be quantified by the maximum nondimensional impulse, $\bar{I}_{\max }=I_{\max } / I_{0}$, applied on the load surface of the structure.

The analytical solutions for the nondimensional impulse $\bar{I}^{(k)}$ of three substructures agree well with the FE predictions and Taylor's solutions (see Figure 15). As the thickness of the structure increases, the plate takes longer time to reach its maximum nondimensional impulse. This is due to the fact that the larger thickness of the structure will prolong the propagating time and thus release higher energy to the plate. The comparisons of the analytical results and FE predictions of maximum nondimensional impulse $\bar{I}_{\max }^{(k)}$ for the three substructures are presented in Table 2. We can see that the numerical predictions of $\bar{I}_{\max }^{(k)}$ are little smaller than the

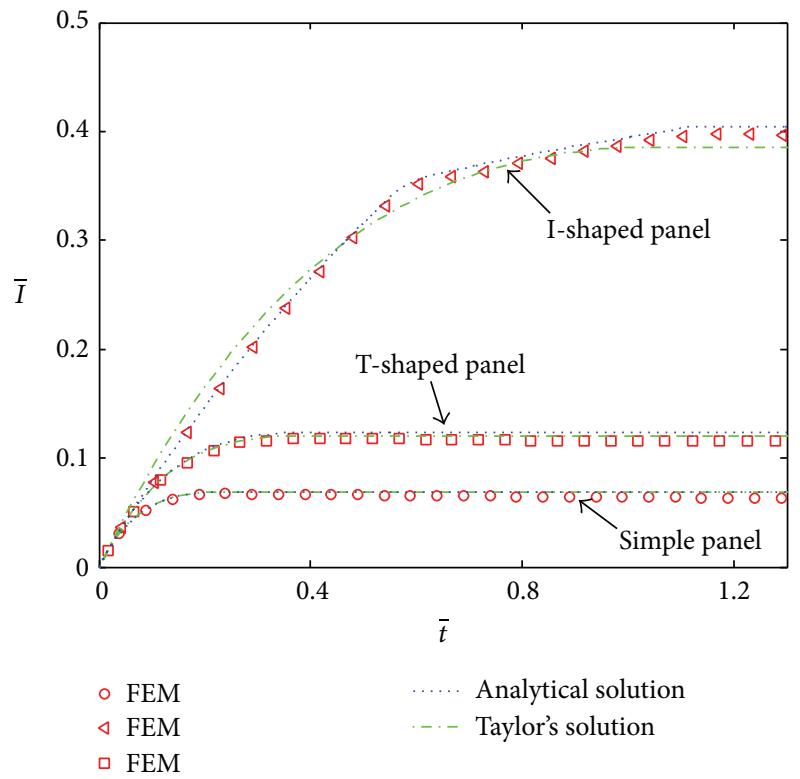

FIGURE 15: The analytical solutions and FE predictions of the nondimensional impulse for (a) simple panel; (b) the panel with stiffener (T-shaped); (c) the panel with girder (I-shaped).

analytical results. This is probably caused by the numerical dissipation. As shown in the second column of Table 2, the normalized impulses $\bar{I}_{\max }^{(k)}$ for the three types of plates equal to $6.81 \%, 12.17 \%$, and $39.87 \%$, respectively, and are all lower than unity. Moreover, comparison between the three plates shows that the specific impulse applied on the wet surface of the panel with girder (I-shaped) is larger than that of the panel with stiffener (T-shaped) and the simple panel, indicating that 
TABLE 2: Analytical and FE predictions of normalized impulse.

\begin{tabular}{lccc}
\hline Case & Analytical results & FE predictions & Error \\
\hline $\begin{array}{l}\text { Simple panel } \\
\begin{array}{l}\text { The panel with } \\
\text { stiffener (T-shaped) }\end{array}\end{array}$ & 0.0681 & 0.0673 & $1.25 \%$ \\
$\begin{array}{l}\text { The panel with girder } \\
\text { (I-shaped) }\end{array}$ & 0.1217 & 0.1184 & $2.71 \%$ \\
\hline
\end{tabular}

more momentum is transmitted to the panel with girder (Ishaped). This is due to the fact that the pressure on the wet surface of the panel with girder (I-shaped) decreases slowly and therefore the impulse impacting the plate will increase.

\section{Sensitivity of the Response to Nondimensional Geometric Parameters}

Having established the accuracy of the analytical predictions, the analytical models are now used to determine the sensitivity of the plate response to the nondimensional parameters of $\bar{\alpha}^{(k)}=l_{f} / t_{m}, \bar{\beta}^{(k)}=d_{m} / t_{f}$, and $\bar{\gamma}^{(k)}=l_{b} / t_{m}$ for the panel with stiffener (T-shaped) and the panel with girder (Ishaped), where the superscript $(k)(k=1,2,3)$ represents the simple panel, the panel with stiffener (T-shaped), and the panel with girder (I-shaped), respectively.

4.1. Response of the Panel with Stiffener (T-Shaped). $\bar{\alpha}^{(2)}$ is the basic parameter of the panel with stiffener (T-shaped). In this case, to investigate the effect of the single parameter $\bar{\alpha}^{(2)}$ on the design of the panel with stiffener (T-shaped), we set $\bar{\beta}^{(2)}=7$. The time histories of nondimensional wet pressure $\bar{p}_{\text {wet }}^{(2)}$ of different $\bar{\alpha}^{(2)}$ are presented in Figure 16 . For a special case of when $\bar{\alpha}^{(2)}$ is 1 , the problem is the same as the response for the simple panel with a sum thickness of mediums $S_{1}$ and $S_{2}$. In this case, only one characteristic is shown, namely, the drops at specific time ticks. When $\bar{\alpha}^{(2)}$ is greater than 1, the characteristics described in Section 3.2.2 for the panel with stiffener (T-shaped) are shown. When $\bar{\alpha}^{(2)}$ is less than 10 , we find that increasing $\bar{\alpha}^{(2)}$ will lead to larger drops of the pressure, as a result of the smaller decay constant and the shortened cavitation time on the wet surface. When $\bar{\alpha}^{(2)}$ is greater than 10 , there is little difference between time histories of pressure with different $\bar{\alpha}^{(2)}$. When $\bar{\alpha}^{(2)} \rightarrow \infty$, the nondimensional histories of pressure approach those of the simple panel. This can be explained as follows: as $\bar{\alpha}^{(2)}$ increases the corresponding specific mass (per unit area) decreases dramatically and approaches the specific mass of simple panel, which greatly weakens the effect of the stiffener on the associated simple panel.

In Figure 17, the analytical results of nondimensional maximum impulse $\bar{I}_{\max }^{(2)}$ are plotted against the normalized parameters $\bar{\alpha}^{(2)}$ for the panel with stiffener (T-shaped). Taylor's predictions for rigid plates of mass equal to this case are also plotted in Figure 17. Both results predict a similar trend. The results show that $\bar{\alpha}^{(2)}$ increases rapidly with reduction of $\bar{I}_{\max }^{(2)}$. We note that, for $\bar{\alpha}^{(2)} \gg 10$, rate of change of $\bar{I}_{\max }^{(2)}$ with $\bar{\alpha}^{(2)}$ slows down, and the value of $\bar{I}_{\max }^{(2)}$ drops to 0.15 , which is approximately the same value as $\bar{I}_{\max }^{(1)}$ for simple panel. According to the results plotted in Figures 16 and 17, we can conclude that the effect of the stiffener on the associated simple panel is significant only in a very small region $\left(\bar{\alpha}^{(2)}<20\right)$. To enhance the significant effect of stiffeners on the associated panel, we can add the number of stiffeners with the same panel.

To investigate the effect of the single parameter $\bar{\beta}^{(2)}$ on the dynamic response of the panel with stiffener (T-shaped) we set $\bar{\alpha}^{(2)}=15$. Figure $18(\mathrm{a})$ presents the dependence of $\bar{p}_{\text {wet }}^{(2)}$ upon $\bar{\beta}^{(2)}$. According to the times the input is wave transmitted through the panel with stiffener (T-shaped) (specific time ticks), the time histories of nondimensional pressure can be divided into few phases. As $\bar{\beta}^{(2)}$ increase, the time the wave travels through T-shaped once increases linearly. That is, the time period between two time ticks increases as $\bar{\beta}^{(2)}$ increase. The trends in each phase are similar and the plate with small $\bar{\beta}^{(2)}$ is easier to get cavitated. Figure 18 (b) shows the sensitivity of the nondimensional impulse $\bar{I}_{\text {max }}^{(2)}$ to the normalized parameter $\bar{\beta}^{(2)}$. The analytical solution and Taylor's solution have the same trend. The $\bar{I}_{\max }^{(2)}$ increases linearly with $\bar{\beta}^{(2)}$. It means that the larger time period between two time ticks can enlarge the impulse on the wet surface. The trend is the consequence of the fact that the ability of the panel with stiffener ( $\mathrm{T}$ shaped) to transport energy increases with increasing $d_{m}$. Therefore, to enhance the effect of stiffeners on the associated plate, another available way is to increase the value of $\bar{\beta}^{(2)}$.

4.2. Response of the Panel with Girder (I-Shaped). Figure 19 shows that the nondimensional pressure histories for parameter $\bar{\alpha}^{(3)}$ for some values between 1 and 70. In these cases, $\bar{\gamma}^{(3)}$ equals 2 and $\bar{\beta}^{(3)}$ equals 80 . It is found that the nondimensional time histories of pressure on the wet surface can be divided into three phases. In the first phase (before the rarefaction wave travels back to the wet surface), the pressure linearly decreases with increasing $\bar{\alpha}^{(3)}$. In the second phase (after the rarefaction wave reaches the wet surface and before cavitation occurs), the pressure drops sharply; we name it the "cut-off" effect. When $\bar{\alpha}^{(3)}$ is less than 10 , we can observe that the "cut-off" effect is more and more significant as $\bar{\alpha}^{(3)}$ increases. When $\bar{\alpha}^{(3)}$ is greater than 10 , the "cut-off" effect directly leads to cavitation on the wet surface. The phenomenon of second phase can be explained as follows: due to increasing $\bar{\alpha}^{(3)}$, the corresponding solid mass (per unit area) decreases, and thus the velocity of wet surface gets larger, resulting in cavitation. Thus, the pressure on the wet surface decreases quickly. In the third phase (after the cavitation occurs), we can see that the cavitation time is shortened when $\bar{\alpha}^{(3)}$ increases.

By integrating the pressure histories, we can obtain the analytical maximum nondimensional impulse (see 


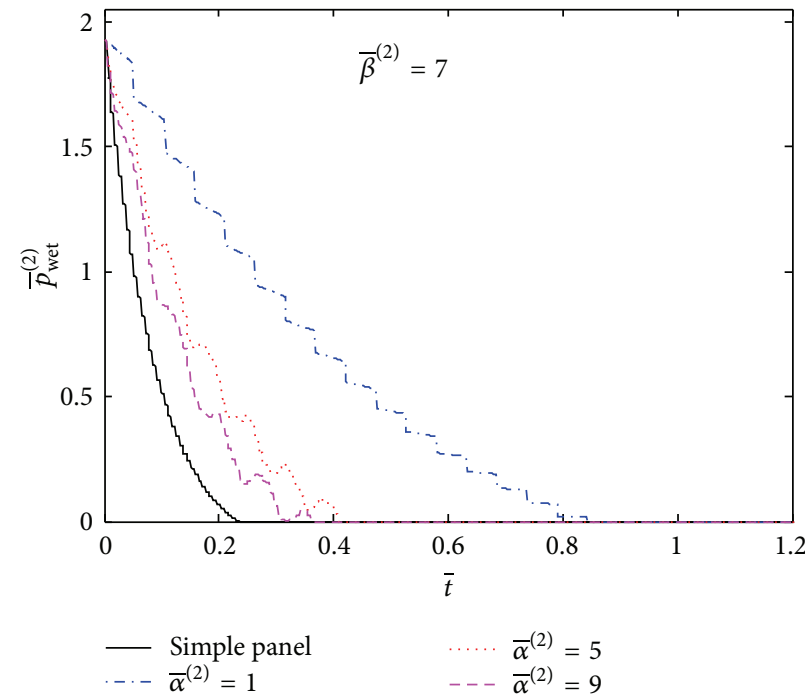

(a)

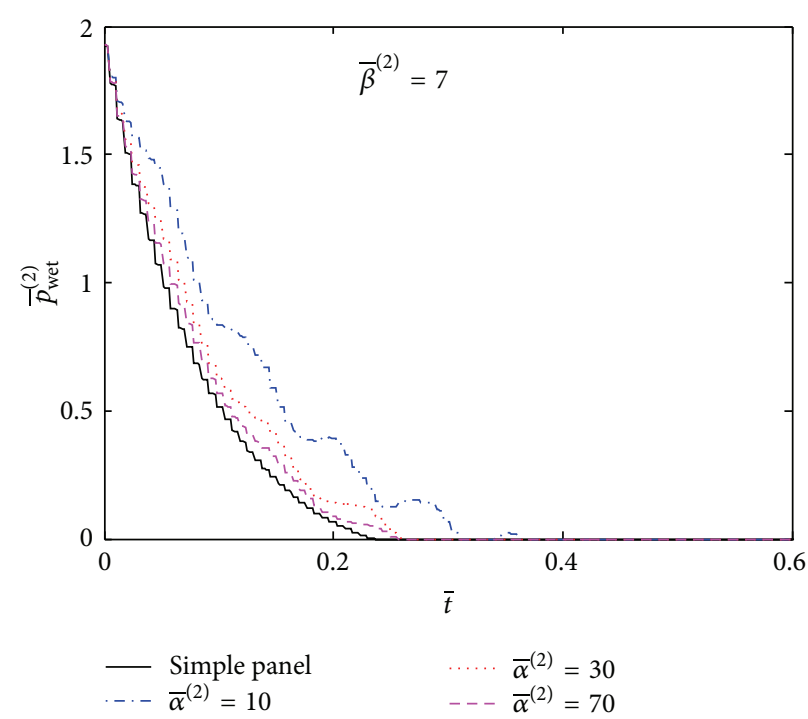

(b)

FIGURE 16: The nondimensional time histories of the nondimensional pressure $\bar{p}_{\text {wet }}^{(2)}$ for the panel with stiffener (T-shaped) with the parameters (a) $\bar{\alpha}^{(2)}=1 \sim 10, \bar{\beta}^{(2)}=7$; (b) $\bar{\alpha}^{(2)}=10 \sim 100, \bar{\beta}^{(2)}=7$.

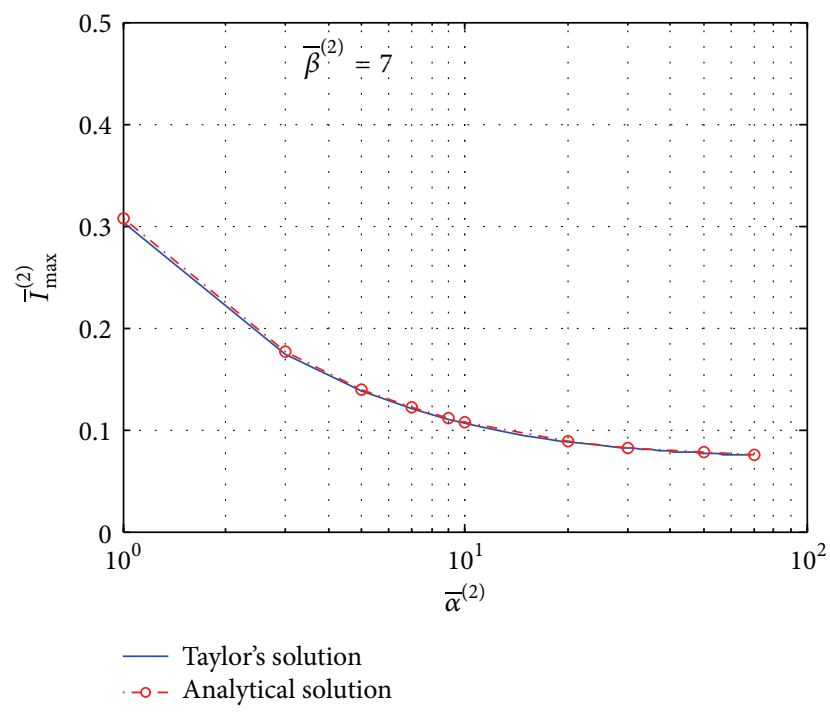

FIGURE 17: Sensitivity of the nondimensional impulse $\bar{I}_{\max }^{(2)}$ to the nondimensional parameter $\bar{\alpha}^{(2)}$ of the panel with stiffener (Tshaped).

Figure 20). It is noted that when $\bar{\alpha}^{(3)}$ is less than 3 , the nondimensional impulse is greater than unity. This is because the total impulse on the wet surface is $2 p_{0} \theta$ for the stationary rigid plate case. Therefore, for the heavy plates, the velocity is small and results in $I /\left(2 p_{0} \theta\right)$ higher than 0.5 . Similarly, the significant effect of girders on the associated panel is limited to the parameter $\bar{\alpha}^{(3)}$. When $\bar{\alpha}^{(3)}>10$, the maximum impulse exponentially decays.

As shown in Figure 21(a), the $\bar{\gamma}^{(3)}$ is set to be 3 , and $\bar{\alpha}^{(3)}$ is 7. The time histories of nondimensional pressure $\bar{p}_{\text {wet }}^{(3)}$ still can be taken as three phases. The phases are divided based on the time the input wave travels once through the panel with girder (I-shaped). As $\bar{\beta}^{(3)}$ increases, the time for the wave to propagate once through the panel with girder (I-shaped) increases linearly. The trends in the whole process with different $\bar{\beta}^{(3)}$ are the same and share similar characteristics: the great drop occurs at the specific time tick, small oscillations occur between time ticks, and there is a little jump due to medium $S_{3}$. Looking at the results plotted in Figures 19 and 21(a), we found out that $\bar{\alpha}^{(3)}$ plays a key role in the first phase and $\bar{\beta}^{(3)}$ determines the time ticks of the phase. In the third phase, we see that the plate with small $\bar{\beta}^{(3)}$ is easier to get cavitated on wet surface. The corresponding maximum impulse $\bar{I}_{\max }^{(3)}$ is shown in Figure 21(b). The impulse is approximately linear with increasing $\bar{\beta}^{(3)}$, indicating that large $\bar{\beta}^{(3)}$ increases the effect of girders on the associated panel.

To investigate the effect of $\bar{\gamma}^{(3)}$, we set $\bar{\alpha}^{(3)}$ equal to 7 and $\bar{\beta}^{(3)}$ equal to 80 . The pressure histories for various $\bar{\gamma}^{(3)}$ are shown in Figure 22(a). As we can see, $\bar{\gamma}^{(3)}$ has a key effect on the jump of the pressure time histories. This stress enhancement occurs when it comes to large impedance ratio on the interface. When the wave transmits from medium $S_{2}$ to medium $S_{3}$, due to the varying section, a strengthened reflected wave is generated on the interface of medium $S_{2}$ and medium $S_{3}$. And this strengthened reflected wave reaches the wet surface before the rarefaction wave from the rear surface. As such, an interesting phenomenon occurs; there are small jumps before the great drop in pressure, except for the case of $\bar{\gamma}^{(3)}=1$. When $\bar{\gamma}^{(3)}=1$, the problem becomes the same as that used to solve the response for the panel with stiffener (T-shaped). In this case, the small jump 


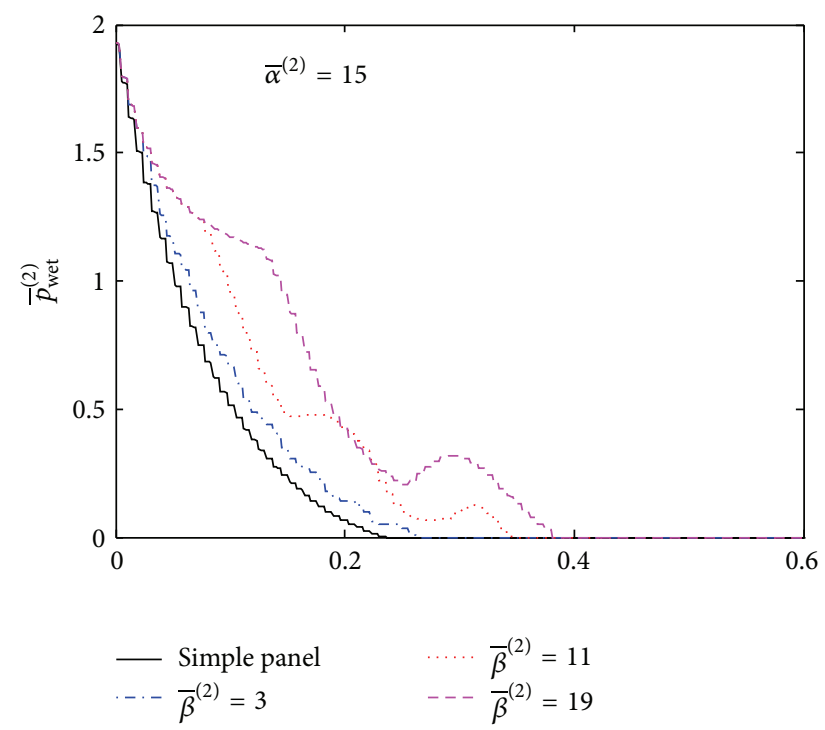

(a)

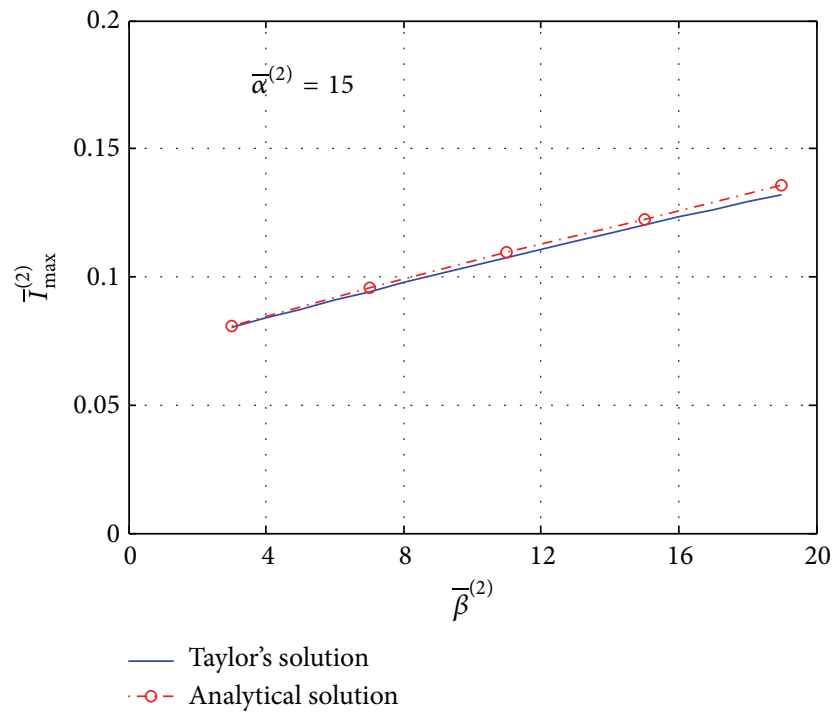

(b)

FIGURE 18: (a) The normalized time histories of the nondimensional pressure $\bar{p}_{\text {wet }}^{(2)}$ for the panel with stiffener (T-shaped) with the parameters $\bar{\beta}^{(2)}$ and $\bar{\alpha}^{(2)}=15$; (b) sensitivity of the nondimensional impulse $\bar{I}_{\max }^{(2)}$ to the normalized parameter $\bar{\beta}^{(2)}$ for the panel with stiffener (T-shaped), $\bar{\alpha}^{(2)}=15$.

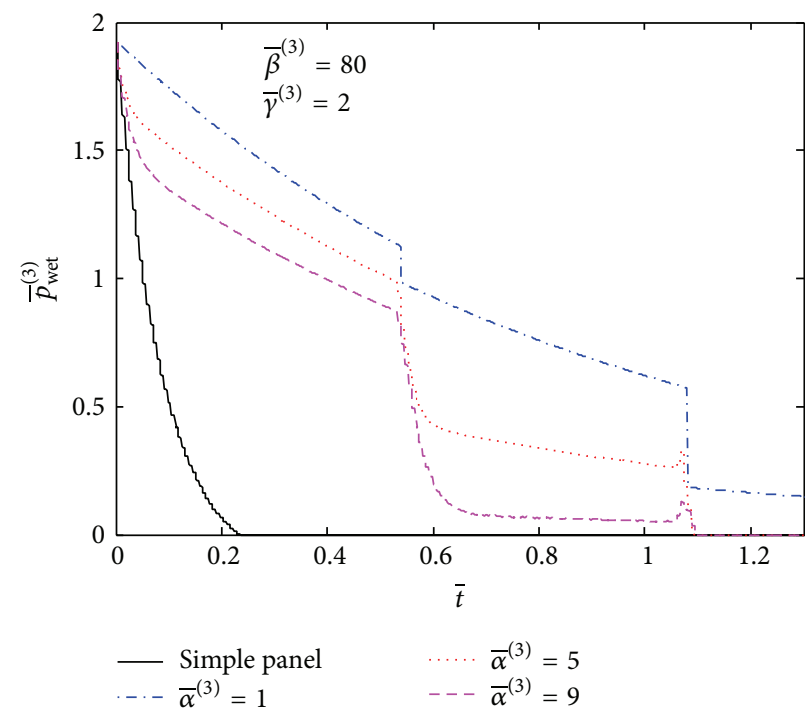

(a)

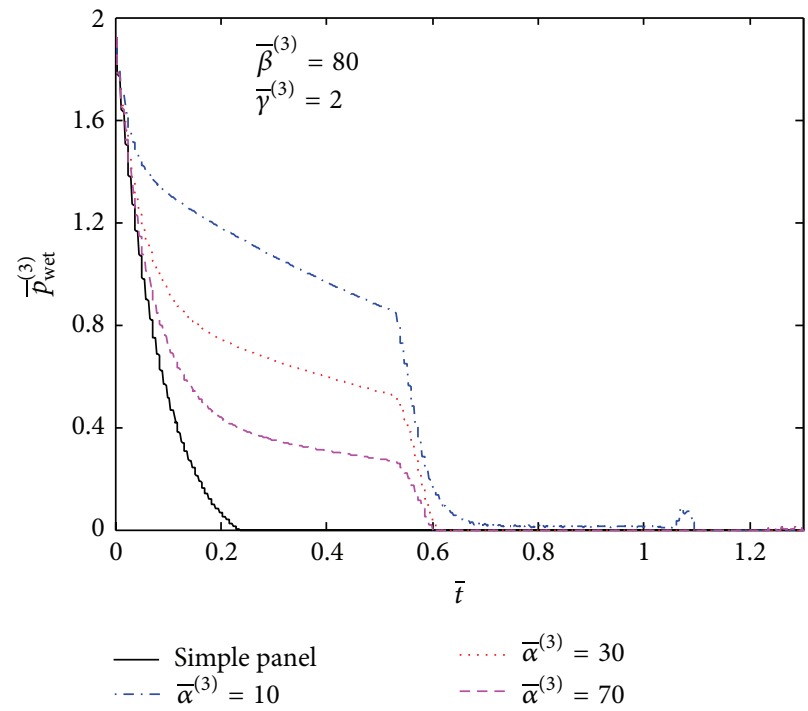

(b)

FIGURE 19: The normalized time histories of the nondimensional pressure $\bar{p}_{\text {wet }}^{(3)}$ for the panel with girder (I-shaped) with the parameter zone (a) $\bar{\alpha}^{(3)}=1 \sim 10, \bar{\beta}^{(3)}=80$, and $\bar{\gamma}^{(3)}=2$; (b) $\bar{\alpha}^{(3)}=10 \sim 70, \bar{\beta}^{(3)}=80$, and $\bar{\gamma}^{(3)}=2$.

before the big drop in time histories of pressure would never happen. From Figure 22(a) it can be seen that the jump is more prominent with the increasing $\bar{\gamma}^{(3)}$. In Figure 22(b), the nondimensional impulse $\bar{I}_{\max }^{(3)}$ is only weakly sensitive to variations of $\bar{\gamma}^{(3)}$.

Taylor's predictions for monolithic plates of equivalent mass are also shown in Figures 20, 21(b), and 22(b). When $\bar{\alpha}^{(3)}<3$ and $\bar{\alpha}^{(3)}>10$, the differences between the analytical solution and Taylor's prediction are prominent. Due to the fact that the strengthened reflected wave in the panel with girder (I-shaped) will cause small jumps in time history of pressure, the nondimensional impulse $\bar{I}_{\max }^{(3)}$ of analytical solutions is slightly higher than these of Taylor's predictions. However, they share a similar trend. 


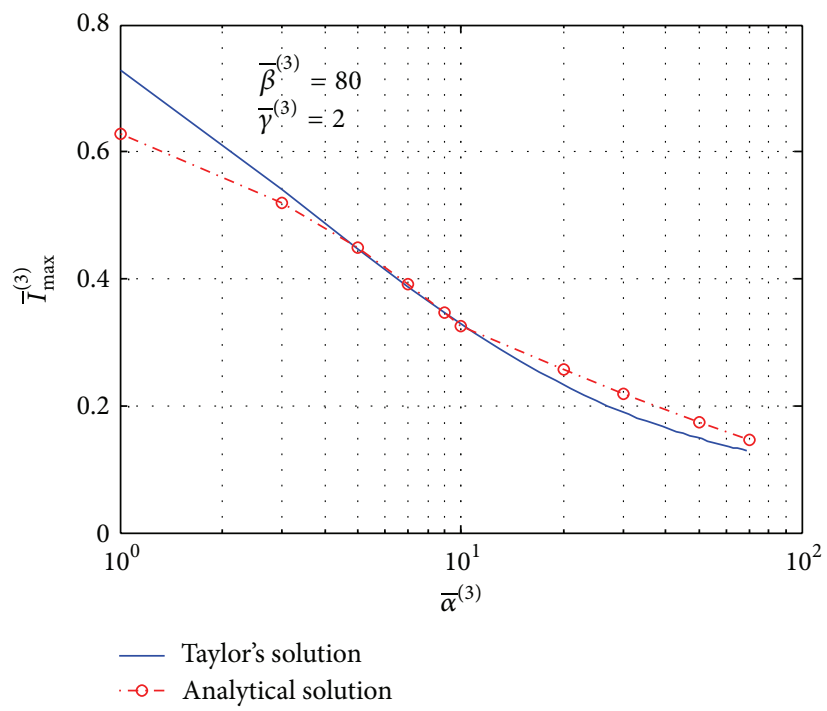

FIGURE 20: Sensitivity of the nondimensional impulse $\bar{I}_{\max }^{(3)}$ to the normalized parameter $\bar{\alpha}^{(3)}$ for the panel with girder (I-shaped).

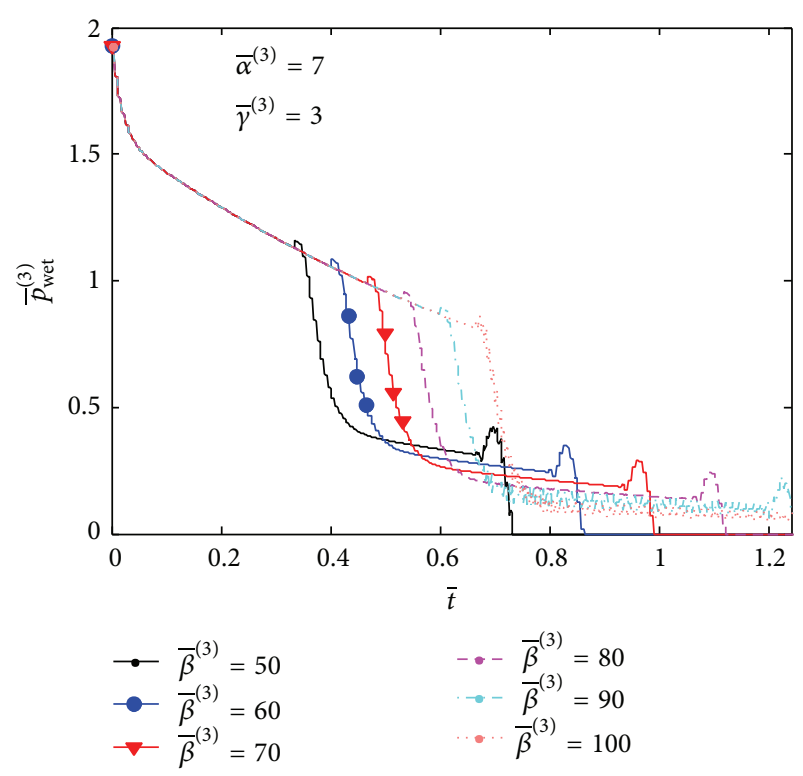

(a)

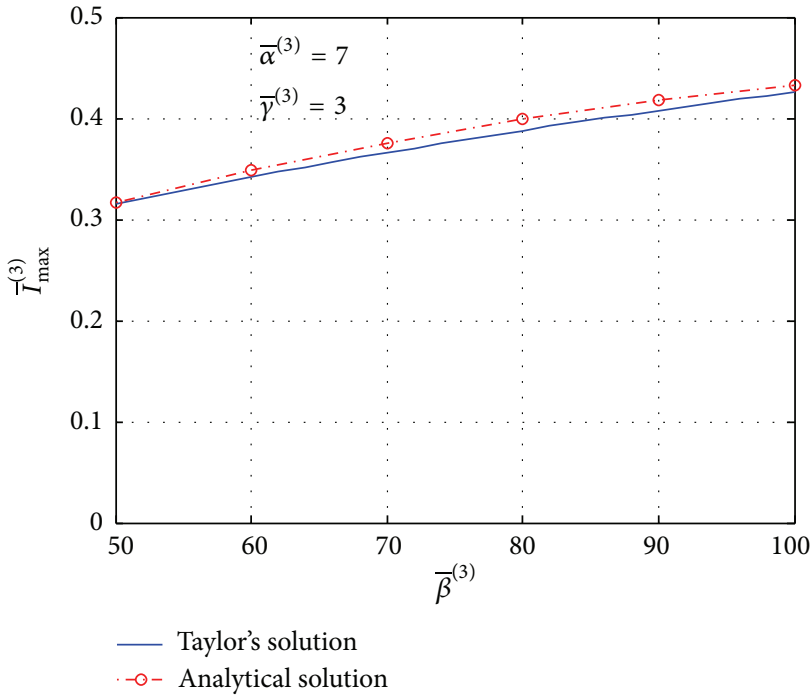

(b)

FIGURE 21: (a) The normalized time histories of the nondimensional pressure $\bar{p}_{\text {wet }}^{(3)}$ for the panel with girder (I-shaped) with the parameter $\bar{\beta}^{(3)}$. (b) Sensitivity of the nondimensional impulse $\bar{I}_{\max }^{(3)}$ to the normalized parameter $\bar{\beta}^{(3)}$ for the panel with girder (I-shaped).

\section{The Interaction between a Double-Bottom Structure and Its Substructures}

In Section 3, we verified the analytical model for the substructures of the double-bottom structure: the simple panel, the panel with stiffener (T-shaped), and the panel with girder (I-shaped). In Section 4, we demonstrated the effects of the nondimensional geometric parameters on the substructures. In this section, we conduct a case study of the substructures, by analytical solution and FE simulation, to obtain the approximate analytical solution to predict the impulse for the complex double-bottom structure system. That is, based on the results from FE method, we try to figure out the interaction between the substructures and a double-bottom structure by using the nondimensional parameters $\bar{\alpha}^{(k)}, \bar{\beta}^{(k)}$, and $\bar{\gamma}^{(k)}$.

It is noted that the parameter $\bar{\alpha}^{(k)}$ for the panel with stiffener (T-shaped) and the panel with girder (I-shaped) is 


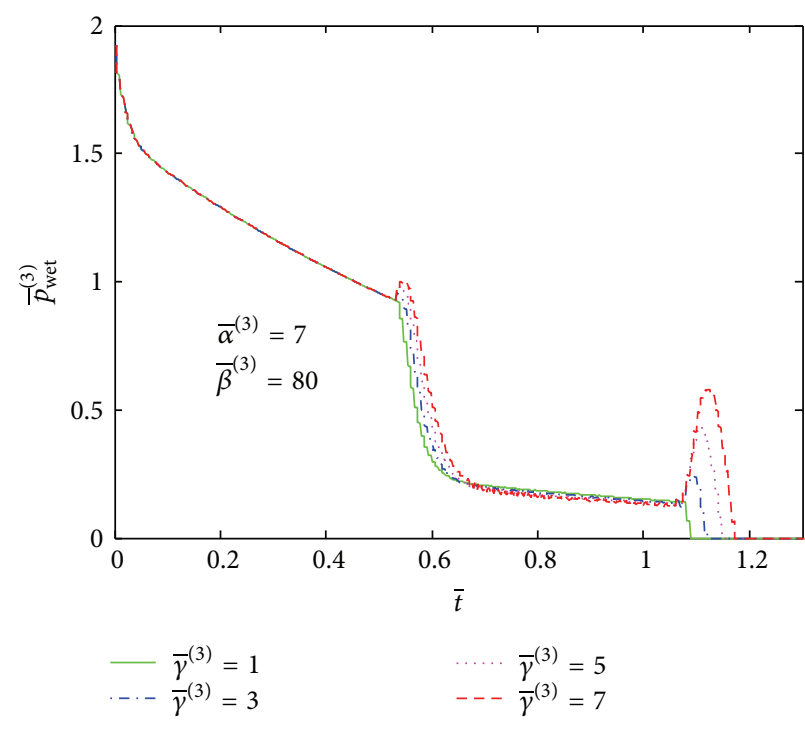

(a)

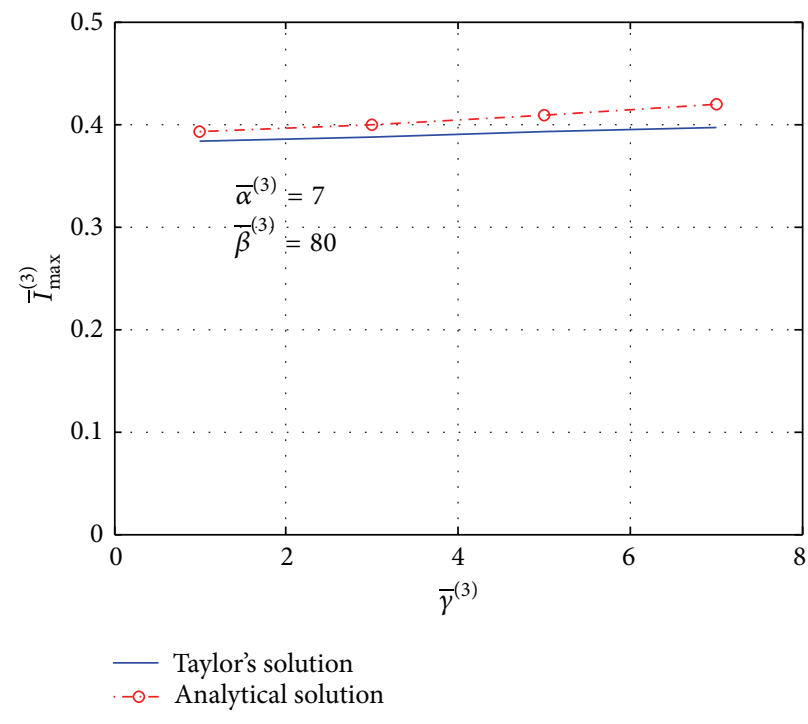

(b)

FIGURE 22: (a) The normalized time histories of the nondimensional pressure $\bar{p}_{\text {wet }}^{(3)}$ for the panel with girder (I-shaped) with the parameter $\bar{\gamma}^{(3)}=1 \sim 10$. (b) Sensitivity of the nondimensional impulse $\bar{I}_{\max }^{(3)}$ to the normalized parameter $\bar{\gamma}^{(3)}$ for the panel with girder (I-shaped).

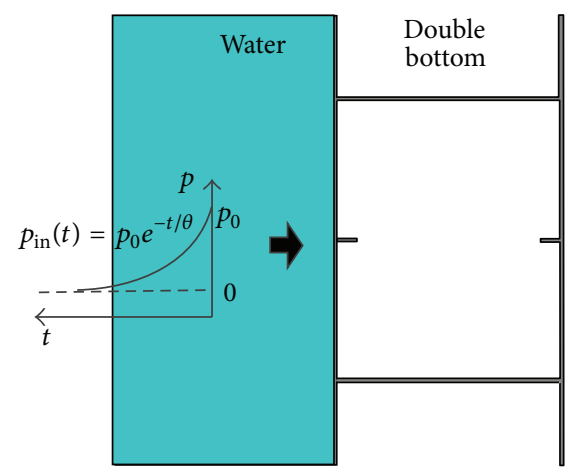

FigURE 23: The sketch of the double-bottom structure cell subjected to underwater explosion.

key to relate the response of the double-bottom structure and the substructures, which can be expressed as

$$
\begin{aligned}
& \bar{I}_{D} \\
& =\frac{\left[\bar{I}^{(1)} \cdot\left(S_{\Gamma}-\sum_{k=2}^{3} \Psi\left(\bar{\alpha}^{(k)}\right)\right)+\sum_{k=2}^{3} \bar{I}^{(k)} \cdot \Psi\left(\bar{\alpha}^{(k)}\right)\right]}{S_{\Gamma}},
\end{aligned}
$$

where the superscript $(k)$ represent the substructures: the simple panel, the panel with stiffener (T-shaped), and the panel with girder (I-shaped), respectively. $\Gamma$ represents the wet surface of the double-bottom structure and $S_{\Gamma}$ is the corresponding area. $\Psi\left(\bar{\alpha}^{(k)}\right)$ is the function which depends on the parameter $\bar{\alpha}^{(k)}$. For simplification, we set $\Psi\left(\bar{\alpha}^{(k)}\right)=$ $\bar{\alpha}^{(k)} \cdot N^{(k)}, k=2,3 . N$ is the sum of the corresponding substructures in the double-bottom structure cell. A cell of a double-bottom structure is defined as the structure assembly between two girders (see Figure 23).
To obtain the solution of (27), an analytical solution and FE simulation are carried out. The FE modeling process is the same as in Section 3.1. Considering the double-bottom structure (see Figure 23), the stiffeners and girders have the same value as shown in Table 1 , and the distance between the girders is $1 \mathrm{~m}$. Even though the double-bottom structure is very complex, to validate the effective model, the FE prediction of impulse of double-bottom structure is solved as an average; namely,

$$
\bar{I}_{D}^{\mathrm{FE}}(t)=\frac{\int_{\Gamma} \int_{0}^{t}\left[\bar{p}_{\mathrm{wet}}^{\mathrm{FE}}(\tau)\right] d \tau d s}{2 \int_{\Gamma} d s},
$$

where $\Gamma$ represent the wet surface.

Figure 24(a) shows the comparison between the FE prediction (obtained from (28)) and the analytical solution (obtained from (27)) for the nondimensional impulse maximum $\bar{I}_{D \text {, max }}$. In this figure, the FE prediction is a constant. To get the same value as the FE prediction, we have to change the value of $\bar{\alpha}^{(k)}$ in (27). By comparing the curves in Figure $24(\mathrm{a})$, we can easily solve (27) by setting $\bar{\alpha}^{(k)}=15$. The definition of the impulse of a double-bottom structure is the summation of the product of impulses of the substructures and the area percentage of the substructures in a doublebottom structure. As $\bar{\alpha}^{(k)}$ increases, the area percentage of the T-shaped panel and the I-shaped panel in a doublebottom structure increases. Based on the above factors, in Figure 24(a), as $\bar{\alpha}^{(k)}$ increases, the maximum impulse increases.

By setting $\bar{\alpha}^{(k)}=15$, the nondimensional time histories of impulse are showed in Figure 24(b). The analytical predictions agree well with FE predictions, except for the small difference from time $0.4 \sim 0.8$ of $1.13 \%$. For the difference, 


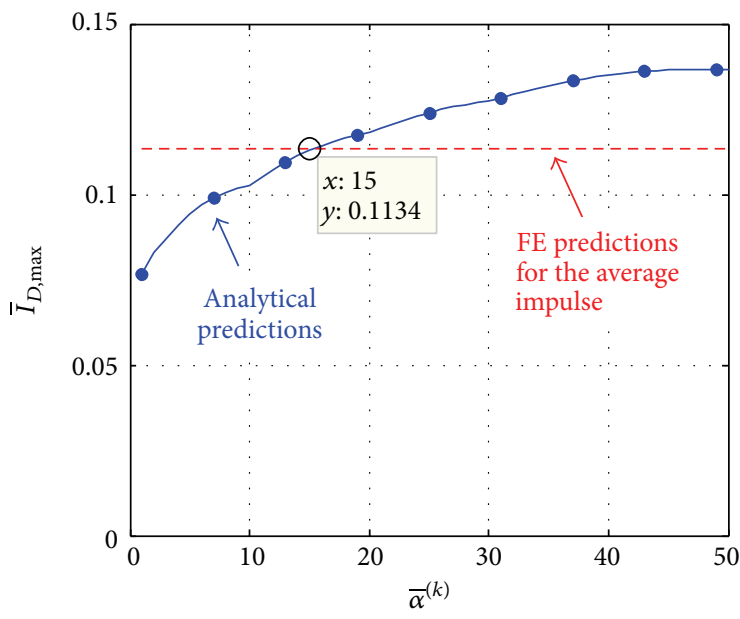

(a)

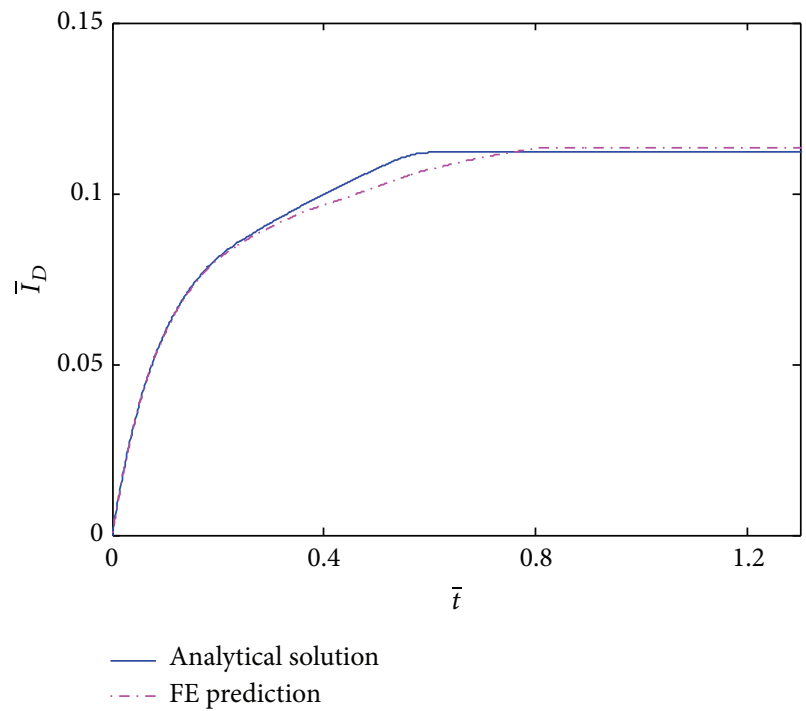

(b)

FIGURE 24: Comparison of analytical solution and FE predictions of (a) the nondimensional maximum impulse and (b) the nondimensional impulse time histories.

the explanations are as follows: the response of the doublebottom is based on the results of substructures; and the cavitation time on the wet surface of substructures is different. In the analytical solution, when cavitation occurs on the wet surface, we assume that the pressure on the wet surface for the corresponding substructures is zero and never changes. After cavitation occurs, the analytical solutions obtained by this assumption cannot present the exact solution in water and thus result in the difference. Actually, the cavitation effect in water is very complex. Compared to the analytical solutions, the FE predictions on water cavitation are more accurate. Though the FE method has the characteristic of being general, the analytical method reveals the underlying physics more clearly and is more convenient to perform a parameter effect analysis. An error of $1.13 \%$ is acceptable, and hence we can still make the conclusion that the approximate analytical solution for double-bottom structure is validated in this case. In other words, when we set $\bar{\alpha}^{(k)}=15$, we can obtain a reasonable impulse for an arbitrary double-bottom structure by (27).

In summary, the steps of the analytical method for an arbitrary double-bottom structure in this paper are as follows: the first step is to divide a double-bottom structure into several substructures according to the nondimensional parameters $\bar{\alpha}^{(k)}$ where $\bar{\alpha}^{(k)}=15$; the second step is to solve the dynamic response of substructures according to the methods in Section 2; the final step is to obtain the dynamic response of double-bottom structure by solving (27) where $\bar{\alpha}^{(k)}=15$.

\section{Conclusion}

In this paper, the response of double-bottom structure to underwater explosion has been investigated by analytical approaches. We have constructed and validated theoretical models for the substructures (the simple panel, the panel with stiffener (T-shaped), and the panel with girder (I-shaped)) loaded by planar, exponentially decaying underwater shock wave. Based on the dynamic response of the substructures, we established the approximate analytical models which are able to predict the response of the double-bottom structure to an underwater explosion. The shock propagation mechanism in the substructures, FSI effects, and cavitation phenomenon were considered in detail. According to the analysis, the main conclusions are as follows:

(1) The analytical models for substructures loaded by exponentially decaying water blast were validated by comparing their predictions to those obtained from fully coupled 3D FE simulations and good agreements were found. However, Taylor's predictions for rigid plates of equivalent areal mass including the effects of cavitation fail to capture the reflected wave from the varying cross section of the structure. It shows a potential application field for the analytical method proposed in this paper.

(2) Writing the expression of dynamic response on the wet surface in nondimensional form shows that the response of substructures to underwater blast depends on three parameters, namely, $\bar{\alpha}^{(k)}, \bar{\beta}^{(k)}$, and $\bar{\gamma}^{(k)} \cdot \bar{\alpha}^{(k)}$ is the key parameter defining the effect of the stiffeners or girders on the associated panel. It shows that the effect of the stiffeners or girders on the associated panel is limited to a small region $\bar{\alpha}^{(k)}<$ 20. The way to enhance this effect is to increase the number of the corresponding substructures. $\bar{\beta}^{(k)}$ is the parameter to prolong the wave transmission time through the structure. Increasing the value of $\bar{\beta}^{(k)}$ is another available way to enhance the effect of the stiffeners or girders on the associated panel. 
(3) $\bar{\alpha}^{(k)}$ is key in relating the response of the substructures and the double-bottom structure. It was shown that by taking $\bar{\alpha}^{(k)}$ as 15 very small difference is found between the analytical solution and the FE prediction. The relationship can be expressed as $\bar{I}_{D}=\left[\bar{I}^{(1)} \cdot\left(S_{\Gamma}-\right.\right.$ $\left.\left.\sum_{k=2}^{3} \Psi\left(\bar{\alpha}^{(k)}\right)\right)+\sum_{k=2}^{3} \bar{I}^{(k)} \cdot \Psi\left(\bar{\alpha}^{(k)}\right)\right] / S_{\Gamma}$. The parameter $\bar{\beta}^{(2)}$ of the panel with stiffener (T-shaped) and $\bar{\beta}^{(3)}$ of the panel with girder (I-shaped) play an important role on FSI dynamic response for double-bottom structure.

The major limitation of the analytical method in the present paper is the assumption that the structure is completely elastic, neglecting plasticity, buckling, and fracture. When a double-bottom structure subjected to underwater blast, the structure would suffer severe deformation. However, the effects of plasticity and buckling cannot be solved by this method. The spherical fashion of the reflected wave at the abrupt change in cross section and the propagation of flexural wave due to bending cannot be captured. Therefore, more detailed calculations of the plastic wave propagation in $2 \mathrm{D}$ and $3 \mathrm{D}$ need to be done to reveal the fluid-structure effect on a double-bottom subjected to underwater explosion. This will from part of our future research.

Despite these limitations, the current double-bottom model and analyses presented in this paper are useful in understanding the fluid-structure interaction, and the elastic stress wave propagation process in complex structures before the impulse cause the bending or the deformation of the whole structure.

\section{Additional Points}

(1) Based on the wave motion theory, we propose analytical models for the simple panel, the panel with stiffener (T-shaped), and the panel associated with girder (I-shaped) to study underwater explosion problem.

(2) Based on the analysis of substructures, we also propose the approximate analytical models for a typical double-bottom structure to investigate underwater explosion problem.

(3) The cavitation effect is taken into consideration to reveal the fluid-structure interaction.

(4) The geometric parameter $\bar{\alpha}^{(k)}$ is key in relating the response of the substructures and the double-bottom structure.

\section{Competing Interests}

The authors declare that there is no conflict of interests regarding the publication of this paper.

\section{Acknowledgments}

The authors thank Dr. Christiaan Adika Adenya for checking the grammar in this paper. The authors also gratefully acknowledge the financial support provided by the National
Natural Science Foundation of China (U1430236, 51479041, 51279038, and 51509228).

\section{References}

[1] A. M. Zhang and Y. L. Liu, "Improved three-dimensional bubble dynamics model based on boundary element method," Journal of Computational Physics, vol. 294, pp. 208-223, 2015.

[2] A. M. Zhang, S. Li, and J. Cui, "Study on splitting of a toroidal bubble near a rigid boundary," Physics of Fluids, vol. 27, no. 6, Article ID 062102, 2015.

[3] A. M. Zhang, P. Cui, J. Cui, and Q. X. Wang, "Experimental study on bubble dynamics subject to buoyancy," Journal of Fluid Mechanics, vol. 776, pp. 137-160, 2015.

[4] A. Schiffer, V. L. Tagarielli, N. Petrinic, and A. C. F. Cocks, "The response of rigid plates to deep water blast: analytical models and finite element predictions," Journal of Applied Mechanics, vol. 79, no. 6, Article ID 061014, 2012.

[5] A.-M. Zhang, L.-Y. Zeng, X.-D. Cheng, S.-P. Wang, and Y. Chen, "The evaluation method of total damage to ship in underwater explosion," Applied Ocean Research, vol. 33, no. 4, pp. 240-251, 2011.

[6] Y. Y. Chen, X. Yao, X. Wei, and X. D. Liu, "Shock response of ship section to underwater explosion with the cavitation effect," in Proceedings of the ASME 33rd International Conference on Ocean, Offshore and Arctic Engineering (OMAE '14), San Francisco, Calif, USA, June 2014.

[7] F. R. Ming, A. M. Zhang, Y. Z. Xue, and S. P. Wang, "Damage characteristics of ship structures subjected to shockwaves of underwater contact explosions," Ocean Engineering, vol. 117, pp. 359-382, 2016.

[8] G. I. Taylor, "The pressure and impulse of submarine explosion waves on plates," in The Scientific Papers of Sir Georey Ingram Taylor, vol. 3, pp. 287-303, Cambridge University Press, Cambridge, UK, 1963.

[9] N. A. Fleck and V. S. Deshpande, "The resistance of clamped sandwich beams to shock loading," Journal of Applied Mechanics, vol. 71, no. 3, pp. 386-401, 2004.

[10] Y. Chen, Y. Wang, and H. X. Hua, "An analytic model for the water blast response of one-dimensional marine structure coated with elastic foam," Archive of Applied Mechanics, vol. 80, no. 11, pp. 1243-1253, 2010.

[11] C. Yin, Z. Jin, Y. Chen, and H. Hua, "One-dimensional response of single/double-layer cellular cladding to water blast," International Journal of Impact Engineering, vol. 88, pp. 125-138, 2016.

[12] W. Xiao, X.-L. Yao, and J. Guo, "Wall effect of underwater explosion load based on wave motion theories," China Ocean Engineering, vol. 28, no. 5, pp. 587-598, 2014.

[13] M. S. H. Fatt and L. Palla, "Analytical modeling of composite sandwich panels under blast loads," Journal of Sandwich Structures and Materials, vol. 11, no. 4, pp. 357-380, 2009.

[14] Z. Jin, C. Yin, Y. Chen, X. Huang, and H. Hua, "An analytical method for the response of coated plates subjected to onedimensional underwater weak shock wave," Shock and Vibration, vol. 2014, Article ID 803751, 13 pages, 2014.

[15] Z.-Y. Jin, C.-Y. Yin, Y. Chen, X.-C. Huang, and H.-X. Hua, "Time-domain analytical solution of one-dimensional wave propagating through underwater multilayer mediums," Journal of Vibration and Shock, vol. 33, no. 13, pp. 6-12, 2014.

[16] E. H. Kennard, "Cavitation in an elastic liquid," Physical Review, vol. 63, no. 5-6, pp. 172-181, 1943. 
[17] A. Schiffer and V. L. Tagarielli, "One-dimensional response of sandwich plates to underwater blast: fluid-structure interaction experiments and simulations," International Journal of Impact Engineering, vol. 71, pp. 34-49, 2014.

[18] A. Schiffer and V. L. Tagarielli, "The one-dimensional response of a water-filled double hull to underwater blast: experiments and simulations," International Journal of Impact Engineering, vol. 63, pp. 177-187, 2014.

[19] A. Schiffer and V. L. Tagarielli, "The response of circular composite plates to underwater blast: experiments and modelling," Journal of Fluids and Structures, vol. 52, pp. 130-144, 2015.

[20] Z. Jin, C. Yin, Y. Chen, and H. Hua, "One-dimensional analytical model for the response of elastic coatings to water blast," Journal of Fluids and Structures, vol. 59, pp. 37-56, 2015.

[21] V. S. Deshpande and N. A. Fleck, "One-dimensional response of sandwich plates to underwater shock loading," Journal of the Mechanics and Physics of Solids, vol. 53, no. 11, pp. 2347-2383, 2005.

[22] J. W. Hutchinson and Z. Xue, "Metal sandwich plates optimized for pressure impulses," International Journal of Mechanical Sciences, vol. 47, no. 4-5, pp. 545-569, 2005.

[23] V. S. Deshpande, A. Heaver, and N. A. Fleck, "An underwater shock simulator," Proceedings of the Royal Society A: Mathematical, Physical and Engineering Sciences, vol. 462, no. 2067, pp. 1021-1041, 2006.

[24] Y. Liang, A. V. Spuskanyuk, S. E. Flores et al., "The response of metallic sandwich panels to water blast," Journal of Applied Mechanics, vol. 74, no. 1, pp. 81-99, 2007.

[25] R. M. McMeeking, A. V. Spuskanyuk, M. Y. He, V. S. Deshpande, N. A. Fleck, and A. G. Evans, "An analytic model for the response to water blast of unsupported metallic sandwich panels," International Journal of Solids and Structures, vol. 45, no. 2, pp. 478-496, 2008.

[26] S. Zhang, "Plate tearing and bottom damage in ship grounding," Marine Structures, vol. 15, no. 2, pp. 101-117, 2002.

[27] L. Hong and J. Amdahl, "Rapid assessment of ship grounding over large contact surfaces," Ships and Offshore Structures, vol. 7, no. 1, pp. 5-19, 2012.

[28] L. L. Wang, Foundations of Stress Waves, Elsevier, 2007.

[29] L. L. Wang, H. S. Bao, and W. X. Lu, "The dependence of adiabatic shear banding on strain-rate, strain and temperature," Journal de Physique, vol. 49, no. 9, pp. 207-214, 1988.

[30] J. M. Boteler and G. T. Sutherland, "Tensile failure of water due to shock wave interactions," Journal of Applied Physics, vol. 96, no. 11, pp. 6919-6924, 2004.

[31] Abaqus, Analysis User's Manual 6.8, 2008. 


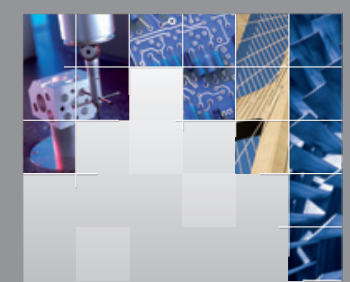

\section{Enfincering}
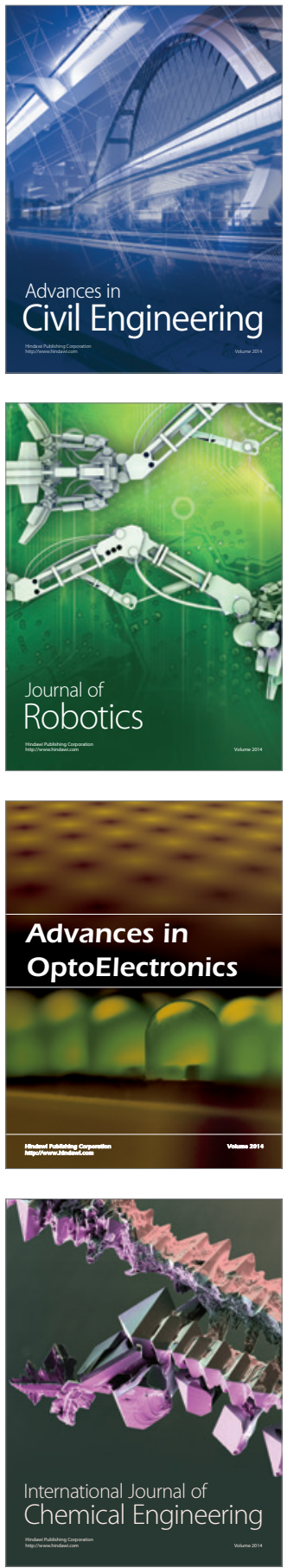

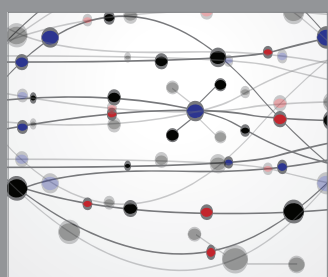

The Scientific World Journal

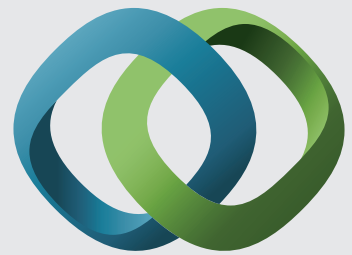

\section{Hindawi}

Submit your manuscripts at

http://www.hindawi.com
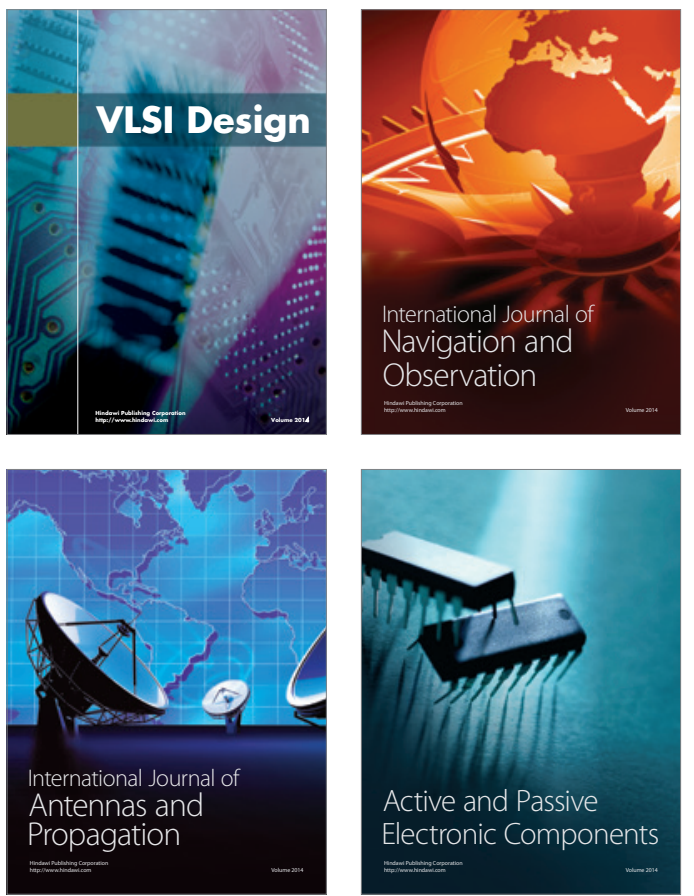
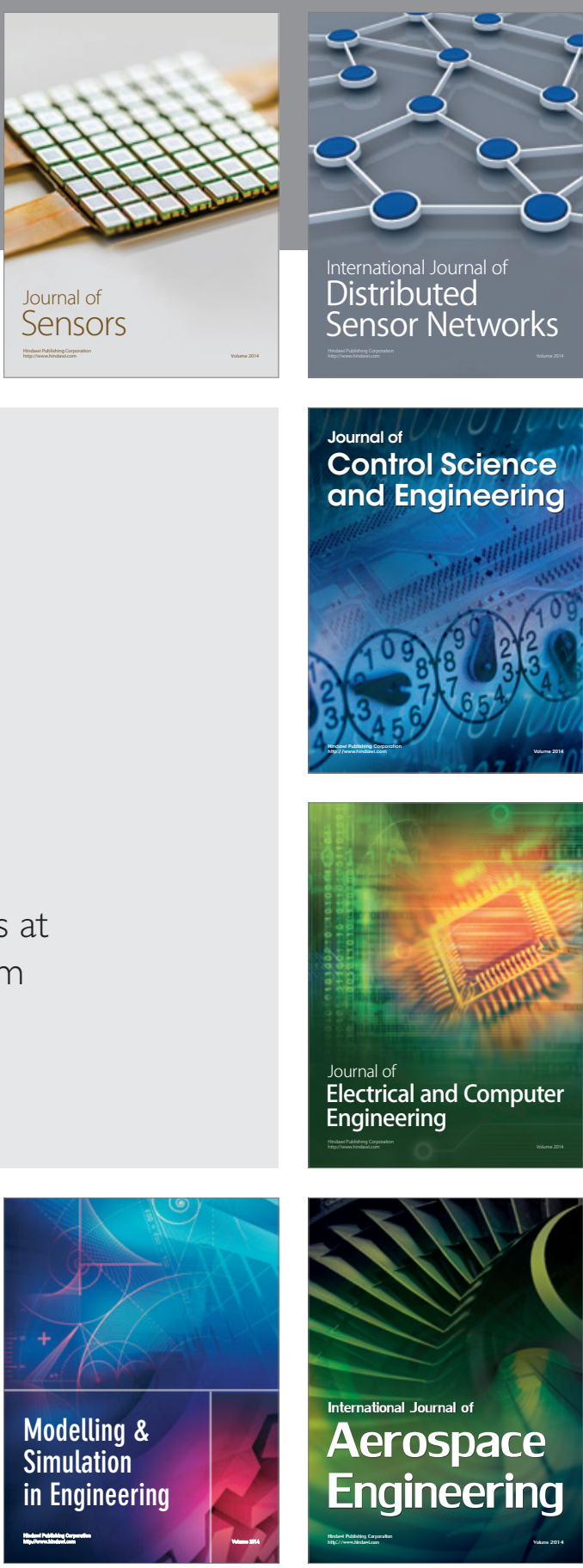

International Journal of

Distributed

Sensor Networks

Journal of

Control Science

and Engineering
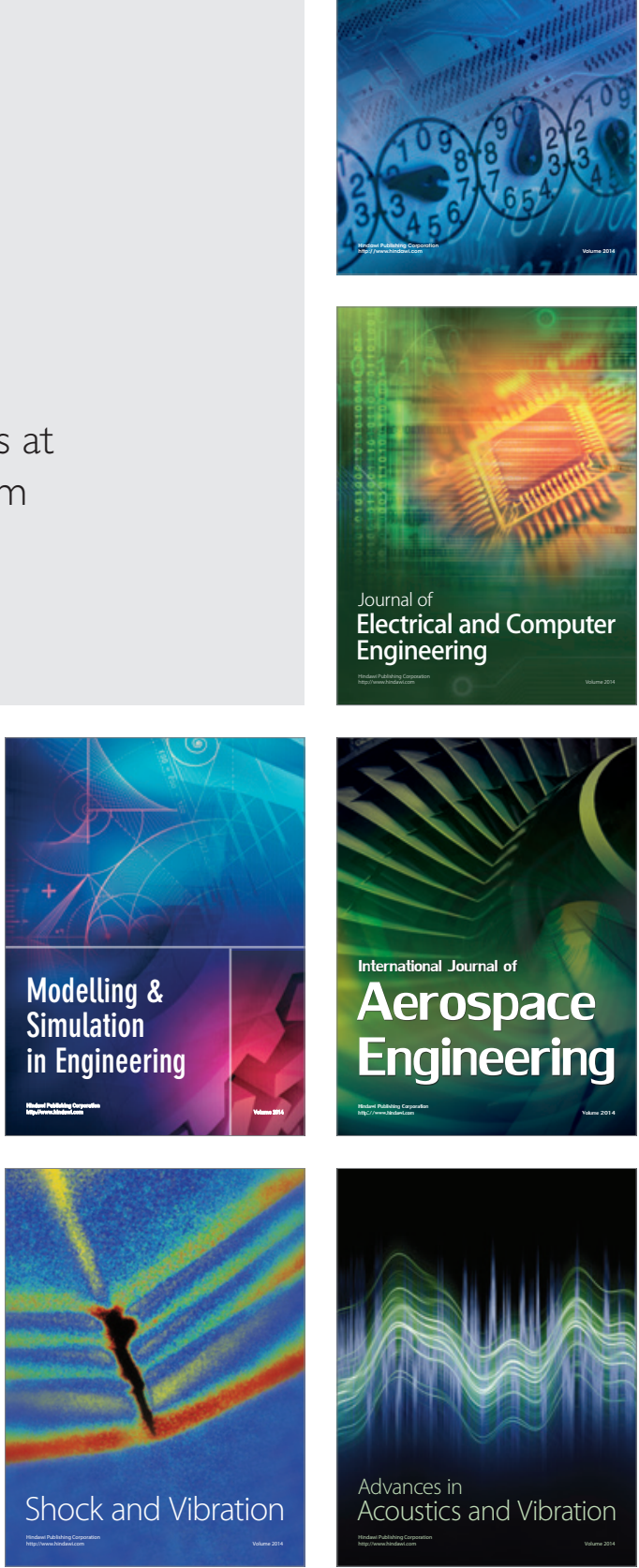